\title{
A Facile Route to Bespoke Macro- and Mesoporous Block Copolymer Microparticles
}

\author{
Guping He, ${ }^{1}$ Thomas M. Bennett, ${ }^{1}$ Mohammad Alauhdin, ${ }^{1}$ Michael W.
} Fay, ${ }^{2}$ Xin Liu, ${ }^{3}$ Simon T. Schwab, ${ }^{1}$ Cheng-gong Sun, ${ }^{3}$ Steven M. Howdle*,1

${ }^{1}$ School of Chemistry, University of Nottingham, Nottingham, NG7 2RD, United Kingdom

${ }^{2}$ Nanoscale and Microscale Research Centre, University of Nottingham, Nottingham, NG7 2RD, United Kingdom

${ }^{3}$ Faculty of Engineering, University of Nottingham, Nottingham, NG7 2RD, United Kingdom

\begin{abstract}
We report a facile and versatile strategy for the bespoke fabrication of macro- and mesoporous block copolymer microparticles. A clean synthetic route, RAFT dispersion polymerisation in supercritical carbon dioxide $\left(\mathrm{scCO}_{2}\right)$, is used to generate block copolymer microparticles. Selective swelling/deswelling is then applied to induce controlled morphology transitions and to trap the resulting porous state. The pore sizes are controllable over a large size range $(\sim 20-200 \mathrm{~nm})$ by varying the length of the swellable block. Through a systematic approach we then demonstrate that the shape of the pores can also be tailored from isolated spheres through to interconnected/bicontinuous channels by varying the ratio of the two blocks. This process is shown to be applicable to a range of poly(methyl methacrylate) (PMMA)based block copolymer systems, including PMMA-b-poly(4-vinyl pyridine), PMMA$b$-poly(dimethyl acrylamide) (DMA) and PMMA- $b$ poly(dimethylaminoethylmethacrylate) (DMAEMA). In each case, the second minority block (e.g. P4VP, etc.) was selectively swollen with an alcohol to induce an order-toorder morphology transition and then quenched rapidly by the non-solvent hexane. This not only takes place on the order of hours, but is also freely scalable for the production of grams of material and beyond in a single step following polymerisation.
\end{abstract}




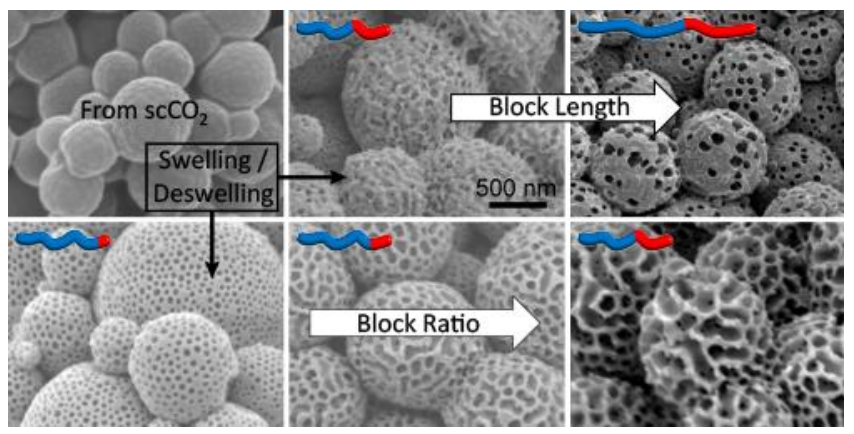

Keywords: porous microparticles, selective swelling, fast de-swelling, controlled pore size, mesopores and macropores, block copolymers.

\section{Introduction}

Porous polymer particles have attracted significant attention because of their potential applicability to mainstream applications such as ion-exchange resins, ${ }^{1,2}$ catalytic supports, ${ }^{3-7}$ chromatography column materials, ${ }^{8,9}$ and as solid supports for organic synthesis. ${ }^{10,11}$ Additionally, there are potentially important emerging applications as templates for materials synthesis, ${ }^{12,}{ }^{13}$ scaffolds for tissue engineering, ${ }^{14-16}$ or as microsensors ${ }^{17,18}$ and microreactors. ${ }^{19-22}$ The key problem is that there is no simple scalable route to synthesise nanoporous microparticulate polymer materials with tunable dimensions across both length scales.

Block copolymers (BCPs) have been extensively exploited for the preparation of porous materials with well-defined nanopores. ${ }^{23-28}$ Owing to the thermodynamic incompatibility of homopolymer chains, microphase separation occurs to yield periodic domains typically on the scale of $10-100 \mathrm{~nm}$. These are well understood and give characteristic morphologies such as spheres, cylinders, gyroids, and lamellae..$^{29-32}$

The traditional approach to create porous BCPs is to etch away one block to create voids. ${ }^{33}$ Nanoporous polymers generated in this way can be exploited as templates for the formation of other nanostructured materials, for example via sol-gel methods. ${ }^{25}$ The templated materials published so far have pore dimensions ranging from a few to 50 $\mathrm{nm}$ and up until now this has largely been achieved in 2D thin films of up to $500 \mathrm{~nm}$ in thickness. However, the process can be tedious and only a very limited range of BCPs with labile blocks have been devised for such purposes; for example, BCPs containing poly(methyl methacrylate) (PMMA), ${ }^{34,35}$ polylactide, ${ }^{36,37}$ and polymers with double 
bonds, e.g., polybutadiene or polyisoprene, ${ }^{38,39}$ which can be etched away by shortwave UV light exposure, hydrolysis and ozonolysis respectively.

A more recent and simpler approach uses solvent swelling/deswelling and evaporation to generate porosity without the need for sample degradation. The first reports were made on two dimensional (2D) micellar thin films ${ }^{40}$ comprised of various amphiphilic BCPs such as PS- $b$-PAA ${ }^{41-44}$ and polystyrene- $b$-poly(x-vinyl pyridine) (PS- $b$-PxVP, $\mathrm{x}$ $=2^{45-48}$ or $\left.4^{49-51}\right)$. The polar solvents quickly penetrate the thin layers of the minority blocks, causing the micelle cores to undergo swelling that is confined within the continuous phase, while the majority blocks remain in a glassy state. Upon drying, the swollen core-forming chains collapse on the surface of the non-swollen matrix and leave pores centred around the original location of the micelles, hence this method is called "confined swelling-induced pore generation". ${ }^{44,52}$

The same swelling induced porosity strategy has also been successfully utilised for so called one-dimensional (1D) BCP materials such as nanorods and nanofibers. ${ }^{46,}, 53-57$ An additional template of anodic aluminium oxide (AAO) was used to produce nanorods (ca. $500 \mathrm{~nm}$ ) that were then subjected to swelling in a selective solvent, such as ethanol for PS- $b$-P2VP. Compared to 2D thin films, which are confined to a solid substrate on one-side, the nanorods/nanofibers show morphology reconstruction in all directions. ${ }^{46}$, ${ }^{54}$ Moreover, the pores are formed with an internal homogeneous wall/shell structure from the collapsed chains of the minority domains of the BCP. The pore structures were found to depend on the swelling agent, swelling temperature, swelling time, and BCP architecture. ${ }^{46,58,59}$ The pore sizes were all $<50 \mathrm{~nm}$ and the film thickness was limited to about $500 \mathrm{~nm}$. Furthermore, Mei et al. were the first to demonstrate that AAO templates can also be used to produce nanoparticles with various internal nano-scale morphologies, including pores and layers, by varying the length of the swellable block. ${ }^{60}$ Porosity was then introduced into these structures via the same solvent swelling approach.

Another related approach for creating porous nanoparticles is to use BCP solutions to create micelles via precipitation and then to apply a swelling/deswelling process where appropriate. This approach has also been shown to impart control of the pore size, shape and/or the overall particle diameter by varying the properties of the block copolymer 
used. ${ }^{61,62}$ The limitation of this route is that the nanoparticle synthesis requires a slow and controlled precipitation and then evaporation from very dilute solutions ( $\sim 1 \mathrm{wt} . \%)$, a process that cannot easily be scaled to the gram or kilogram scale production of material.

Previously, we reported RAFT controlled dispersion polymerisation in $\mathrm{scCO}_{2}$ as a unique route to create multigram quantities of microparticulate block copolymers. ${ }^{63-65}$ Moreover, we have also demonstrated that the sizes of the microparticles can be controlled reproducibly from $300 \mathrm{~nm}$ through to $5.3 \mu \mathrm{m} .{ }^{66} \mathrm{In}$ this paper we exploit these novel BCP structures to create unique nanoporous microparticles from various systems including PMMA- $b$-P4VP, PMMA- $b$-PDMA and PMMA- $b$-PDMAEMA with controllable porosity over an unprecedented size range (10 $\mathrm{nm}$ to ca. $200 \mathrm{~nm})$. Our methodology exploits synthesis in $\mathrm{scCO}_{2}$ followed by a rapid alcohol based swelling induced morphology reconstruction of the minor block to create voids. To the best of our knowledge, this is the first time nanoporous microparticles with completely bespoke physico-chemical properties have been produced by a method that is realistically scalable.

\section{Experimental Section}

\subsection{Materials}

Methyl methacrylate (MMA, Fisher, >99\%), 4-vinylpyridine (4VP, Acros, 99\%), dimethyl acrylamide (DMA, Aldrich, 99\%), and dimethylaminoethylmethacrylate (DMAEMA, Aldrich, 99\%) were purified by passing through a neutral alumina column and stored at $-20^{\circ} \mathrm{C}$. 2-(dodecylthiocarbonothioylthio)-2-methylpropionic acid (DDMAT) was synthesised following a literature procedure. ${ }^{67} \quad \alpha$ azobis(isobutyronitrile) (AIBN, Wako, 97\%) was purified by recrystallisation in ethanol. The dispersion stabiliser poly(dimethylsiloxane)-monomethyl methacrylate (PDMS-MA, ABCR, $M_{\mathrm{n}}=10,000 \mathrm{~g} / \mathrm{mol}$ ), $\mathrm{CDCl}_{3}$ (Aldrich, 99.9\%), HPLC grade THF (Acros), chloroform (Aldrich, 99.9\%), triethylamine (Acros, 99.9\%) and iodine (Fisher) were used as received. Ruthenium tetroxide solutions were freshly prepared by mixing $12.4 \mathrm{mg}$ of ruthenium chloride $\left(\mathrm{RuCl}_{3}\right.$, Aldrich, $\left.99.9 \%\right)$ and $4.2 \mathrm{mg}$ of sodium periodate ( $\mathrm{NaIO}_{4}$, Aldrich, 99.9\%) in $1 \mathrm{ml}$ deionized $\mathrm{H}_{2} \mathrm{O}$ solution at $0{ }^{\circ} \mathrm{C}$ prior to use. Agar 100 resin (Agar Scientific) was used as received, and a formulation of medium hardness 
was used for embedding samples.

\subsection{Synthesis of BCP microparticles via RAFT controlled dispersion in $\mathrm{scCO}_{2}$}

The synthesis of BCP microparticles followed earlier published procedures. ${ }^{63}, 64 \mathrm{~A}$ typical one-pot synthesis is described below for PMMA50- $b$-P4VP 33 where the subscripts denote the target $\mathrm{MW}$ in $\mathrm{kg} / \mathrm{mol}$. A high-pressure autoclave $(60 \mathrm{ml})$ was flushed with $\mathrm{CO}_{2}(5 \mathrm{MPa})$ for $15 \mathrm{~min}$ before adding the reactants for the synthesis of the first block. MMA (7.5 g), DDMAT RAFT agent (55 mg), AIBN initiator (25 mg), and PDMS-MA surfactant $(0.625 \mathrm{~g})$ were pre-mixed in a glass vial and purged with nitrogen for $20 \mathrm{~min}$ before transferal into the autoclave with a glass syringe. The $\mathrm{CO}_{2}$ pressure in the autoclave was first increased to $\sim 6 \mathrm{MPa}$ and the autoclave was then heated to $60{ }^{\circ} \mathrm{C}$ whilst the contents were stirred mechanically. ${ }^{68}$ The final pressure and temperature were gradually adjusted to $\sim 27 \mathrm{MPa}$ and $65^{\circ} \mathrm{C}$ over a period of $15 \mathrm{~min}$. The reaction was carried out for 18 hours to achieve full conversion of the MMA. The second monomer 4VP (5 g) and additional AIBN (6.3 mg) were purged with $\mathrm{N}_{2}$ for 15 min and added to the autoclave via a HPLC pump at $1 \mathrm{ml} / \mathrm{min}$. The polymerisation of 4VP was then allowed to proceed for $20 \mathrm{~h}$ before the autoclave was first cooled down to $25{ }^{\circ} \mathrm{C}$ and then depressurised. The product was observed to be an off-white fine free flowing dry powder and was collected for analysis.

\subsection{Selective swelling}

To introduce porosity to the as-synthesised $\mathrm{BCP}$ microparticles, the original $\mathrm{BCP}$ microparticles $(50 \mathrm{mg})$ were fully dispersed in alcohol $(3 \mathrm{ml})$ in a glass vial $(3.5 \mathrm{ml})$ and shaken 3 times by hand over a two-hour period. The particles were left standing until they settled to the bottom of the vial. The upper alcohol layer was removed and then hexane $(3 \mathrm{ml})$ was poured into the vial and the particles were fully rinsed by vigorous shaking. The particles were then allowed to resettle before removing the hexane layer after $2 \mathrm{~h}$. The hexane rinse was then repeated three times to completely remove all of the alcohol and then dried in a vacuum oven at $25{ }^{\circ} \mathrm{C}$ for $>2 \mathrm{~h}$ before further analysis.

\subsection{Analysis}

The block copolymers were characterised by ${ }^{1} \mathrm{H}$ NMR in $\mathrm{CDCl}_{3}$ on a Bruker AV3400 
(400 MHz) spectrometer. Gel Permeation Chromatography (GPC) analysis was carried out on an Optilab-rEX (Wyatt) in a mixture of chloroform/ethanol/triethylamine (90/9/1 by volume) for PMMA- $b$-P4VP at a flow rate of $0.5 \mathrm{~mL} / \mathrm{min}$ and $25^{\circ} \mathrm{C}$. GPC Columns were composed of K-G, a K-805L and a HT-803 column (Shodex) and calibrated with PMMA narrow standards.

For SEM, the samples were mounted on an aluminium stub and sputter-coated with platinum or iridium prior to imaging on a JEOL 7100F FEG-SEM at accelerating voltage of $2 \mathrm{kV}$ or a FEI Quanta 650 ESEM at $10 \mathrm{kV}$. The average pore width based on SEM was measured by counting over 100 pores using the commercial software package NanoMeasurer 1.2.5.

Thin sections for cross-sectional TEM were obtained from the original powder BCP microparticle samples by embedding them in an epoxy resin (Agar 100) cured at $55^{\circ} \mathrm{C}$ for 2 days. Thin sections ( $\sim 80 \mathrm{~nm}$ ) were cut by ultra-microtome using a diamond knife (Leica Diatome Ultra $45^{\circ}$ ) and were placed on copper TEM grids (Agar). The porous microparticle samples were similarly mounted but were cut by cryo-ultra-microtome. The samples were first placed on the surface of a drop of sucrose solution (5\%) on a sample stick following by freezing in liquid nitrogen. The frozen microparticles were then ultra-microtomed into thin sections of $\sim 100 \mathrm{~nm}$ at $-60{ }^{\circ} \mathrm{C}$ using a glass knife. These cross-sections on the copper grids were then imaged using either a JEOL 2100Plus TEM at $200 \mathrm{kV}$ or FEI Tecnai BioTwin-12 TEM at $100 \mathrm{kV}$ at room temperature. PMMA- $b$-P4VP samples were stained with $\mathrm{I}_{2}$ vapour for $\sim 2 \mathrm{~h}$ where stated to enhance the domain contrast in the images. P4VP preferentially absorbs $\mathrm{I}_{2}$ and appears as dark regions in bright field TEM. The domain sizes in the TEM were measured by counting over 100 domains using the software package NanoMeasurer 1.2.5.

The porosity and surface area of the prepared samples were characterised with a Micromeritics ASAP 2420 instrument by $\mathrm{N}_{2}$ adsorption at $77 \mathrm{~K}$. Prior to the measurements the samples were first degassed at $70{ }^{\circ} \mathrm{C}$ for $16 \mathrm{~h}$. The surface area was calculated by the Brunauer-Emmett-Teller (BET) method using the $\mathrm{N}_{2}$ adsorption isotherm data within the relative pressure ranging from 0.05 to 0.3 . The density functional theory (DFT) method, which is described in detail elsewhere, ${ }^{69,}{ }^{70}$ was used to extract the pore size distribution from the adsorption branch using the Micromeritics 
software. When both mesopores and macropores exist in a material it is necessary to assess the pore width distribution relative to both pore volume and surface area; mesopores contribute to surface area, whilst macropores significantly increase pore volume.

Tilt TEM and Tomography Conventional bright-field TEM of these particles is limited by two factors, first, that the image is a 2-dimensional projection and second, that the sample has to be thin enough to allow at least partial transmission of the electron beam. The complexity of a fully porous 3-dimensional structure is not readily comprehended in a 2-dimensional projection. However, many of the microparticles could be seen to be partially electron transparent even at their thickest points. Since this allows information to be obtained from the entire volume, and the contrast mechanism of conventional bright-field TEM images in these samples is predominantly proportional to volume, tomographic reconstruction from a tilt series was therefore possible.

For an accurate reconstruction, the sample was deposited onto a lacey carbon support TEM grid pre-prepared with $10 \mathrm{~nm}$ gold fiducial markers to aid computer alignment. A total of 120 images were manually acquired at 1 degree steps in a single axis tilt series, using a Gatan 916 room temperature tomography holder in a JEOL 2100Plus equipped with a Gatan US1000 CCD camera at $200 \mathrm{kV}$. Post-acquisition alignment and reconstruction was performed using the IMOD software (http://bio3d.colorado.edu/imod/) utilising the WBP and SIRT reconstructions.

\section{Results and Discussion}

\subsection{Fabrication of porous microparticles via selective swelling of the minority block}

Poly(methyl methacrylate)- $b$-poly(4-vinyl pyridine) (PMMA- $b$-P4VP) $\left(M_{\mathrm{n}}=56400\right.$ $\mathrm{g} / \mathrm{mol} ; \bigoplus=1.47$ ), denoted as M-V27.8 (where M represents PMMA, V represents P4VP, and 27.8 represents the molar fraction of $\mathrm{P} 4 \mathrm{VP}$ ) was synthesised as microparticles via RAFT controlled dispersion polymerisation in $\mathrm{scCO}_{2}$. After swelling with ethanol, a selective solvent for P4VP, and rinsing with the non-solvent hexane the particle morphology was clearly porous (Fig. 1a). The SEM image shows open surface pores 
that are partially interconnected into sub surface cylindrical pores and a deeper pore network, with an average pore width of $d_{\mathrm{w}}=112.7 \mathrm{~nm}$. The subsurface porosity is demonstrated by SEM images at higher magnification (Fig. 1b) where much smaller pores with $d_{\mathrm{w}}=20.3 \mathrm{~nm}$ are visible below. TEM images (Fig. 1c) indicate that the porosity persists throughout the entire microparticle. A thin slice $(\sim 150 \mathrm{~nm})$ was cut by cryo-ultra-microtome and the TEM images (Fig. 1d) confirm the pores are interconnected and form a bicontinuous porous network.
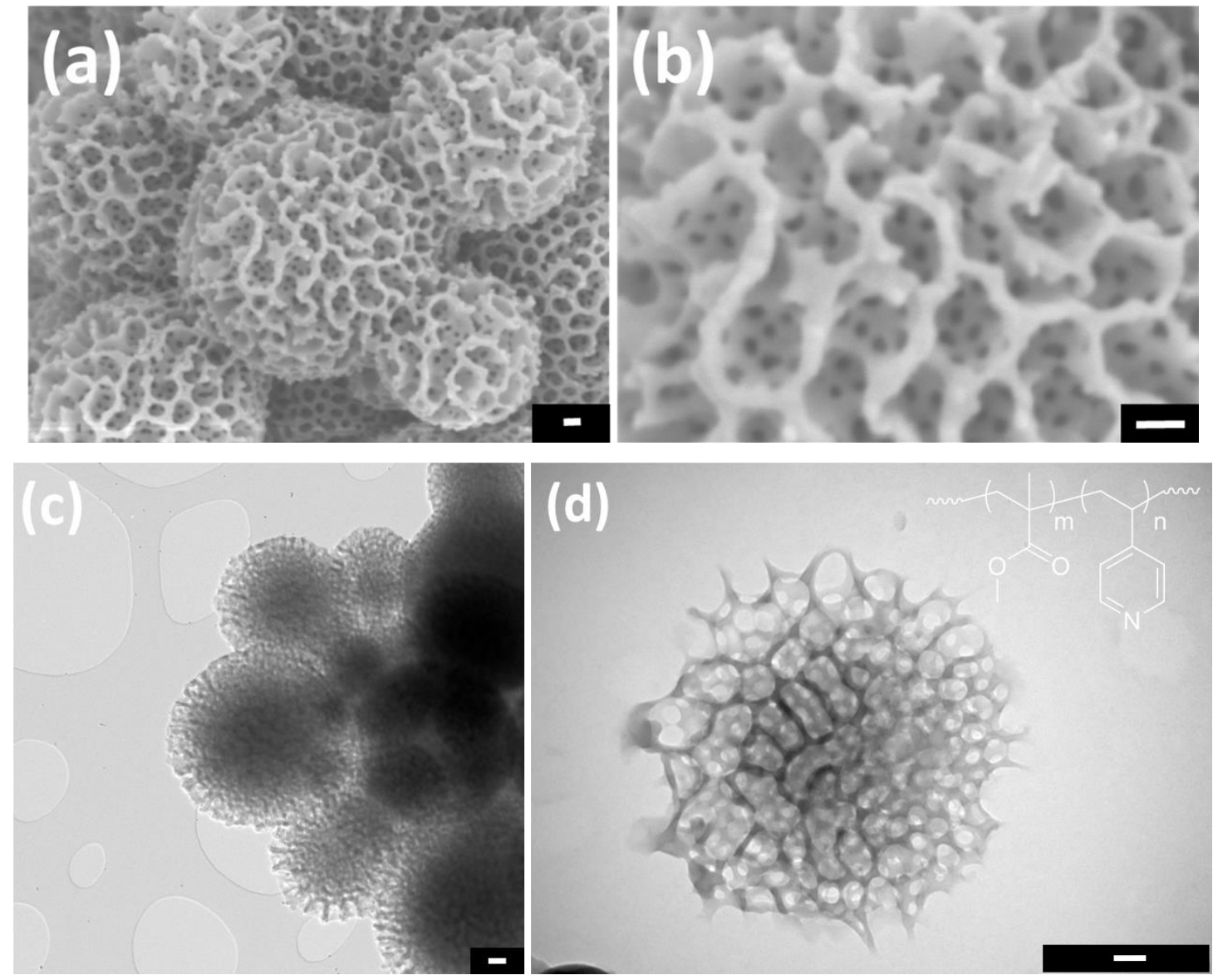

Figure 1. SEM and TEM images of PMMA- $b$-P4VP (M-V27.8) porous microparticles with pores created by selective swelling in ethanol. $(a, b)$ SEM images of the porous microparticles at different magnification at $2 \mathrm{kV}$, (c) particle TEM image at $200 \mathrm{kV}$, (d) cross sectional TEM image of sliced section ( $\sim 150 \mathrm{~nm}$ thickness by cryo-ultra-microtome) without staining and at $100 \mathrm{kV}$. The inset in (d) is the chemical structure of PMMA- $b$-P4VP. Scale bar represents 100 $\mathrm{nm}$ in all cases.

Prior to ethanol swelling the original M-V27.8 microparticles have a phase separated morphology of spherical P4VP domains in a PMMA matrix (Fig. S1c). These internal 
patterns were formed in situ by polymerisation induced phase separation. ${ }^{63}$ The minority P4VP domains show an average size of $d=21.7 \mathrm{~nm}$ (measured from TEM). A distinctive periphery clearly shows larger P4VP domains with $d=60.9 \mathrm{~nm}$ (refer to S2 for all TEM-derived domain size measurements). After the solvent swelling interconnected channels are formed and these have an average internal pore width of $d_{\mathrm{w}}=51.1 \mathrm{~nm}$ (Fig. 1d), which is twice the original P4VP domain size of $d=21.7 \mathrm{~nm}$ and is a result of the P4VP ethanol swelling interaction.

The swelling - deswelling process is best described schematically (Scheme 1). The swelling of these 3D microparticles is somewhat different from 2D thin films, ${ }^{41,44,51,52}$ which generally have one-side confined by a solid substrate and the swollen minority chains can only move towards the free surface. In a 3D BCP microparticle system, the selective solvent penetrates and swells the microparticle from all directions and the movement of the swollen chains as well as the plastic deformation of the non-swollen glassy domains occurs symmetrically. Initially, the spherical domains of the minority component P4VP are swollen with the selective solvent ethanol (Scheme 1a). As its volume increases, the minority component pierces through the glassy domains of the majority block PMMA and a bicontinuous morphology evolves (Scheme 1b). Hexane is a poor solvent for both PMMA and P4VP but it is miscible with ethanol. Upon the addition of hexane the swollen minority block collapses (Scheme 1c), but the nanoporous morphology of the partially swollen BCP microparticles is fixed by the rigid, continuous scaffold that is formed by the glassy PMMA. Thus, nanopores with walls consisting of the collapsed minority P4VP blocks are formed in place of the original P4VP domains (Scheme 1d). Furthermore, the collapse of the surface layer, which largely consists of P4VP, leads to the formation of an open nano-porous system at the microparticle surface. Critically, the high $T_{\mathrm{g} S}$ of both blocks and their inertness to air, humidity and (moderate) temperatures ensures that the resulting porous microparticles are stable at ambient conditions indefinitely (Fig. S3).

(a)

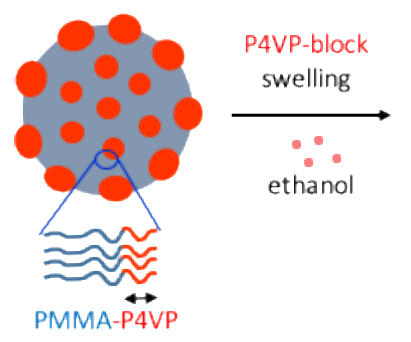

(b)

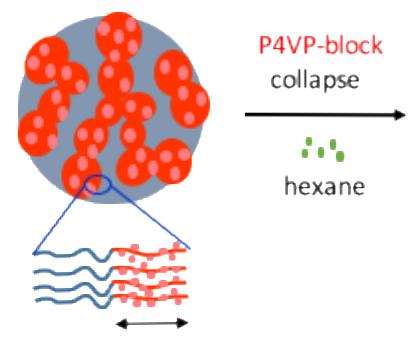

(c)

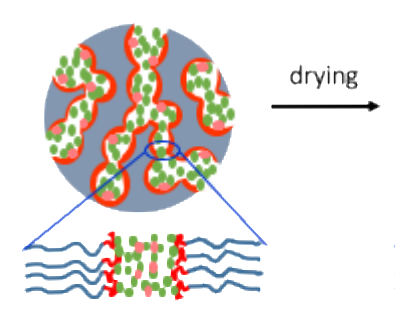

(d)

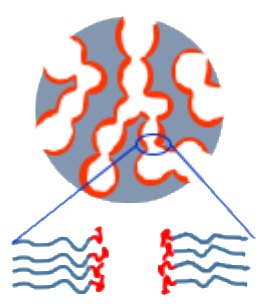


Scheme 1. Schematic demonstration of pore generation throughout BCP microparticles via selective solvent swelling induced order-to-order morphology transition and then morphology fixation by fast de-swelling.

\subsection{Pore size and shape control}

To investigate the effect of the volume fraction of the minority P4VP block on the porosity, we synthesised PMMA- $b$-P4VP microparticles with similar PMMA chain lengths (ca. MW $=\sim 50 \mathrm{~kg} / \mathrm{mol}$ ) and varied the P4VP chain length to give molar fractions from 9 - 45 mol\% (Table 1, refer to Fig. S4 for the raw GPC data). The smallest P4VP block (2.6 kDa, M-V9.1) likely does not phase separate and shows no readily visible structure in the TEM (see Fig. S1e). However, when the P4VP block is a little longer $>13 \mathrm{~mol} \%$ (M-V13.3) the polymeric microparticles show internal phase separation of spherical P4VP domains in a PMMA matrix (TEM mapping of thin sections Fig. S1a). For larger P4VP blocks, the domain size increases from $12.5 \mathrm{~nm}$ to $25.5 \mathrm{~nm}$ as the P4VP chain length increases and the P4VP fraction rises from $13.3 \mathrm{~mol} \%$ to $30.9 \mathrm{~mol} \%$. A distinctive periphery layer of large P4VP domains begins to appear very strongly as the P4VP content is increased to $19 \mathrm{~mol} \%$ (Fig. S1b-d). These peripheral P4VP domains are enlarged from $57 \mathrm{~nm}$ to $106.3 \mathrm{~nm}$ at 19.9 and $30.9 \mathrm{~mol} \%$ P4VP respectively (Fig. S1, 3).

It appears that the peripheral surface structure is likely a consequence of progressive monomer exclusion towards the interior of the microparticles. Therefore, at the latter stages of polymerisation the P4VP domains at the periphery are swollen with excess 4VP monomer, leading to the much larger domains as compared with the interior. We did kinetic studies of the copolymerization via a sampling tap connected to the autoclave as described previously and the corresponding samples were microtomed and investigated by TEM. These images corroborate the 4VP domains become larger at the particle surface towards the latter stages of the reaction (Fig. S5). This also likely accounts for the slightly broader than expected MW distributions ca. 1.4 - 1.5 (Table 1). GPC analysis was used to exclude the possibility of P4VP homopolymer contamination for each sample. In each case no obvious shoulders were present, and extensive washing with ethanol (a good solvent for P4VP) showed no distinct mass loss or GPC profile changes (see Fig. S6 for M-V27.8). 
Table 1. Characterisation of the PMMA- $b$-P4VP block copolymers synthesised via RAFT dispersion polymerisation in $\mathrm{ScCO}_{2}$.

\begin{tabular}{|c|c|c|c|c|c|c|c|c|}
\hline \multirow{3}{*}{$\begin{array}{l}\text { Entries } \\
\text { M-Vx } \\
\end{array}$} & \multirow{3}{*}{ Target $\mathbf{M}_{\mathbf{n}}$} & \multirow{3}{*}{ NMR } & \multicolumn{4}{|c|}{$\mathbf{G P C}^{\mathrm{a}}$} & \multicolumn{2}{|c|}{$M_{n} b y^{1} H N M R^{b}$} \\
\hline & & & \multicolumn{2}{|c|}{$1^{\text {st }}$ block PMMA } & \multicolumn{2}{|c|}{ BCPs } & \multirow{2}{*}{$\begin{array}{c}\text { P4VP } \\
M{ }^{\prime}\end{array}$} & \multirow{2}{*}{$\begin{array}{c}\text { BCPs } \\
M_{\mathrm{n}}^{\prime}\end{array}$} \\
\hline & & & $M_{\mathbf{n}}$ & $\boldsymbol{t}$ & $M_{\mathrm{n}}$ & $\boldsymbol{\theta}$ & & \\
\hline M-V9.1 & $50 \mathrm{k} / 2.6 \mathrm{k}$ & $90.9 / 9.1$ & 48500 & 1.26 & 52000 & 1.52 & 5100 & 53600 \\
\hline M-V13.3 & $50 \mathrm{k} / 5.6 \mathrm{k}$ & $86.7 / 13.3$ & 39000 & 1.25 & 51100 & 1.43 & 6300 & 45300 \\
\hline M-V19.9 & $50 \mathrm{k} / 15 \mathrm{k}$ & $80.1 / 19.9$ & 41300 & 1.23 & 58500 & 1.44 & 10800 & 52100 \\
\hline M-V27.8 & $50 \mathrm{k} / 17 \mathrm{k}$ & $72.2 / 27.8$ & -- & -- & 56400 & 1.47 & -- & -- \\
\hline M-V30.9 & $50 \mathrm{~K} / 20 \mathrm{~K}$ & $69.1 / 30.9$ & 40400 & 1.24 & 59400 & 1.50 & 19000 & 59400 \\
\hline M-V36.4 & $50 \mathrm{k} / 25 \mathrm{k}$ & $63.6 / 36.4$ & -- & -- & 58900 & 1.80 & -- & -- \\
\hline M-V36.9 & $200 \mathrm{k} / 133 \mathrm{k}$ & $63.1 / 36.9$ & 119300 & 1.83 & 153100 & 2.24 & 73300 & 192600 \\
\hline \multicolumn{9}{|c|}{$\begin{array}{l}{ }^{\text {a }} \text { mixed solvent of } \mathrm{CHCl}_{3}+\text { ethanol }+ \text { TEA }(90: 10: 1, \mathrm{v} / \mathrm{v} / \mathrm{v}) \text { as eluent and PMMA as calibration } \\
\text { standards; }{ }^{\mathrm{b}} M_{n} \text { is the MW calculation based on both }{ }^{1} \mathrm{H} \text { NMR (block ratio) and GPC (the } M_{n} \text { of }\end{array}$} \\
\hline \multicolumn{9}{|c|}{$\begin{array}{l}M_{n}^{\prime}(P 4 V P) ;{ }^{\mathrm{c}} \mathrm{M}, \mathrm{V} \text {, and the numerals after } \mathrm{V} \text { denote PMMA, P4VP, and the molar fraction of } \\
\mathrm{P} 4 \mathrm{VP} \text { block, respectively; }{ }^{\mathrm{d}} \text { molar ratio of PMMA/P4VP; for entries M-V27.8 and M-V36.4 the } \\
1^{\text {st }} \text { block was not sampled before adding } 4 \mathrm{VP} \text { to the autoclave. }\end{array}$} \\
\hline
\end{tabular}

The BCP microparticles underwent swelling/deswelling as described and SEM images (Fig. 2) show the porous microparticles obtained from PMMA- $b$-P4VP for a range of P4VP molar fractions. At low P4VP fraction (13.3 mol\% - sample M-V13.3) the images show isolated spherical pores on the particle surface (Fig. 2a, b). These surface pores are arranged in a near hexagonal array. As the molar fraction of P4VP is increased to $19.9 \mathrm{~mol} \%$ (sample M-V19.9), the larger surface open pores partially fuse and where 2 to 4 pores merge, they morph into a short cylindrical surface feature (Fig. 2 c, d). This is a direct result of the increased swollen P4VP volume exerting increased pressure on the glassy PMMA matrix, leading to rupturing and merging of isolated spherical P4VP domains. A similar pore structure evolution from spheres to channels was previously 
observed in the swelling of 1D nanorods at longer swelling times. ${ }^{46,48}$ Some interesting pore geometries have also been reported previously by changing the $\mathrm{BCP}$ composition and hence the swellable block fraction in large compound micelles or nanospheres. ${ }^{46}$, $60-62$

When the minority P4VP block is further increased to $27.8 \mathrm{~mol} \%$ (sample M-V27.8) more ethanol absorbs and the swollen P4VP volume increases concomitantly (Fig. 2e, f), giving interconnected open surface pores and a much larger surface pore width, $d_{\mathrm{w}}$ $=112.7 \mathrm{~nm}$ (compared to M-V19.9, $d_{\mathrm{w}}=74.2 \mathrm{~nm}$ ). Thus, the original partially merged spherical P4VP domains observed in M-V19.9 have extended to form a bicontinuous morphology fixed into the glassy PMMA scaffold (Fig. 2e). Increasing the P4VP block further to $30.9 \mathrm{~mol} \%$ (sample M-V30.9) leads to significantly more surface porosity (Fig. 2g, h) where the surface bicontinuous channels now have an average pore width of $d_{\mathrm{w}}=168.8 \mathrm{~nm}$ from the SEM. Finally, the sample with a P4VP block ratio of 36.4 mol\% (M-V36.4) shows a loss of control and collapse of the surface pores (Fig. S9a, b); Coalescence and fusion leads to less well-defined pores because there is insufficient glassy PMMA present to stabilise and fix the swelling induced morphology. 

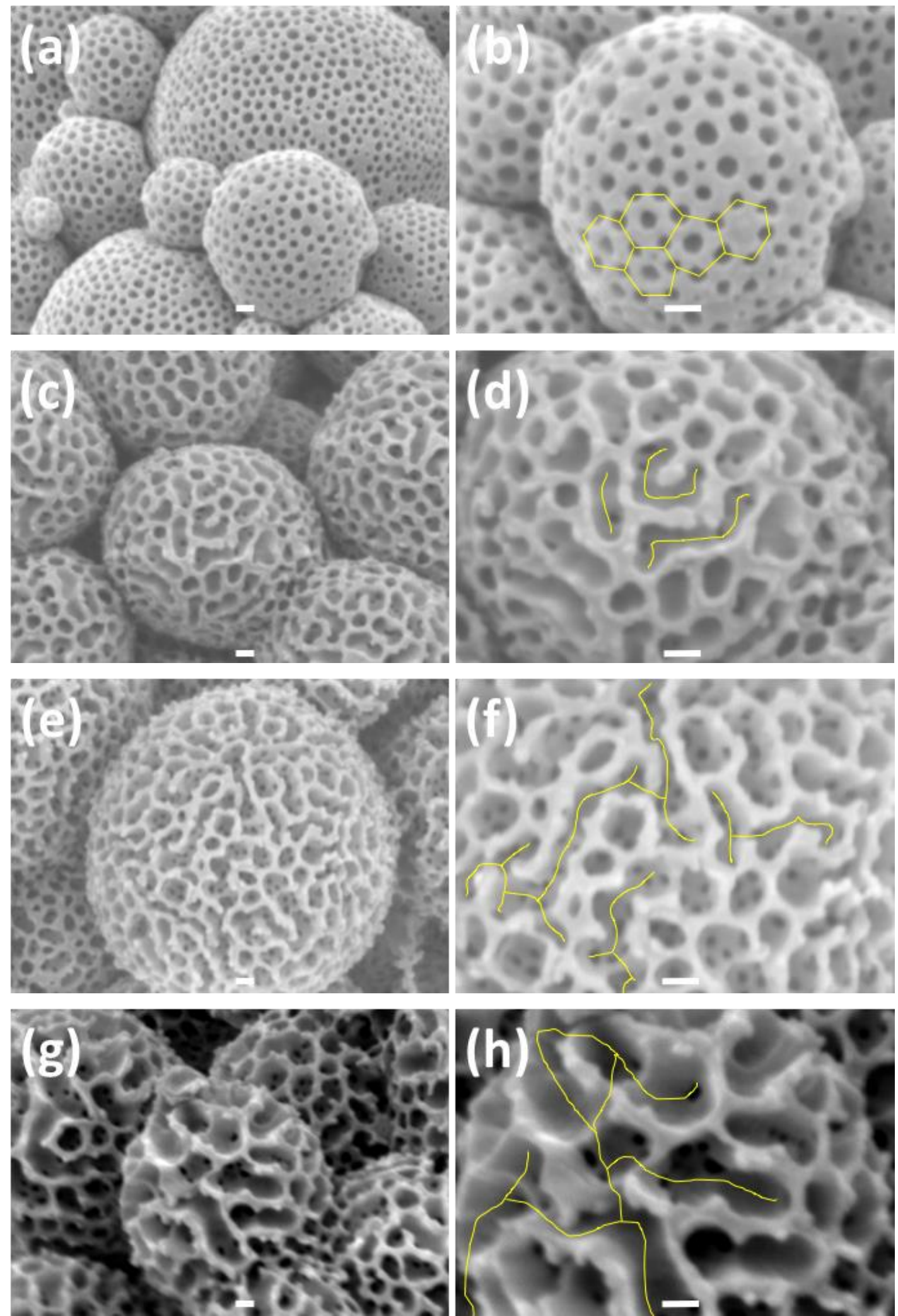

Figure 2. Controlled nanoporosity introduced into block copolymer microparticles by selective swelling/deswelling in ethanol with hexane. SEM images of PMMA- $b$-P4VP porous microparticles. (a) M-V13.3, (c) M-V19.9, (e) M-V27.8, (g) M-V30.9. (b, d, f, h) are higher magnification images of (a, c, e, g) respectively. Scale bar in all cases is $100 \mathrm{~nm}$. The yellow 
lines in $(b, d, f, h)$ mark the surface pore evolution from isolated spherical pores to short channels, and to interconnected channels. The original BCP particles are shown in Fig. S11.

The SEM images also hint at sub-surface pores of the order $d_{\mathrm{w}}=20-25 \mathrm{~nm}$ (Fig. 2); To probe these, the same samples were further characterised by TEM imaging (Fig. 3). For M-V13.3b, the pores only develop near the surface and not throughout the whole particle (Fig. 3a). At higher P4VP content $>19 \mathrm{~mol} \%$, the TEM data clearly demonstrate that the microparticles are highly porous throughout (Fig. 3b-d) and confirm that ethanol diffuses effectively through the glassy PMMA regions, the major component of the BCP microparticles.

We hypothesised that the swelling process for the 3D microparticles is time-dependent. Wang et. al investigated 1D nanorods of PS- $b$-P2VP with ethanol and found that varied pore morphologies were achieved by increasing swelling time from $10 \mathrm{~min}$ to $66 \mathrm{~h} .{ }^{46}$, ${ }^{54}$ However, they began with just a very dilute suspension of nanorods in ethanol and argued that the swelling at $20^{\circ} \mathrm{C}$ was tiny. In our study, the BCP microparticles are a dry powder and we have a much greater material bulk. We found that a substantial time ca. $>1 \mathrm{~h}$ was required just to fully suspend the BCP microparticle powder in ethanol, whereas for full solvent penetration, $>12 \mathrm{~h}$ was required to fully swell gram quantities of our microparticles. 

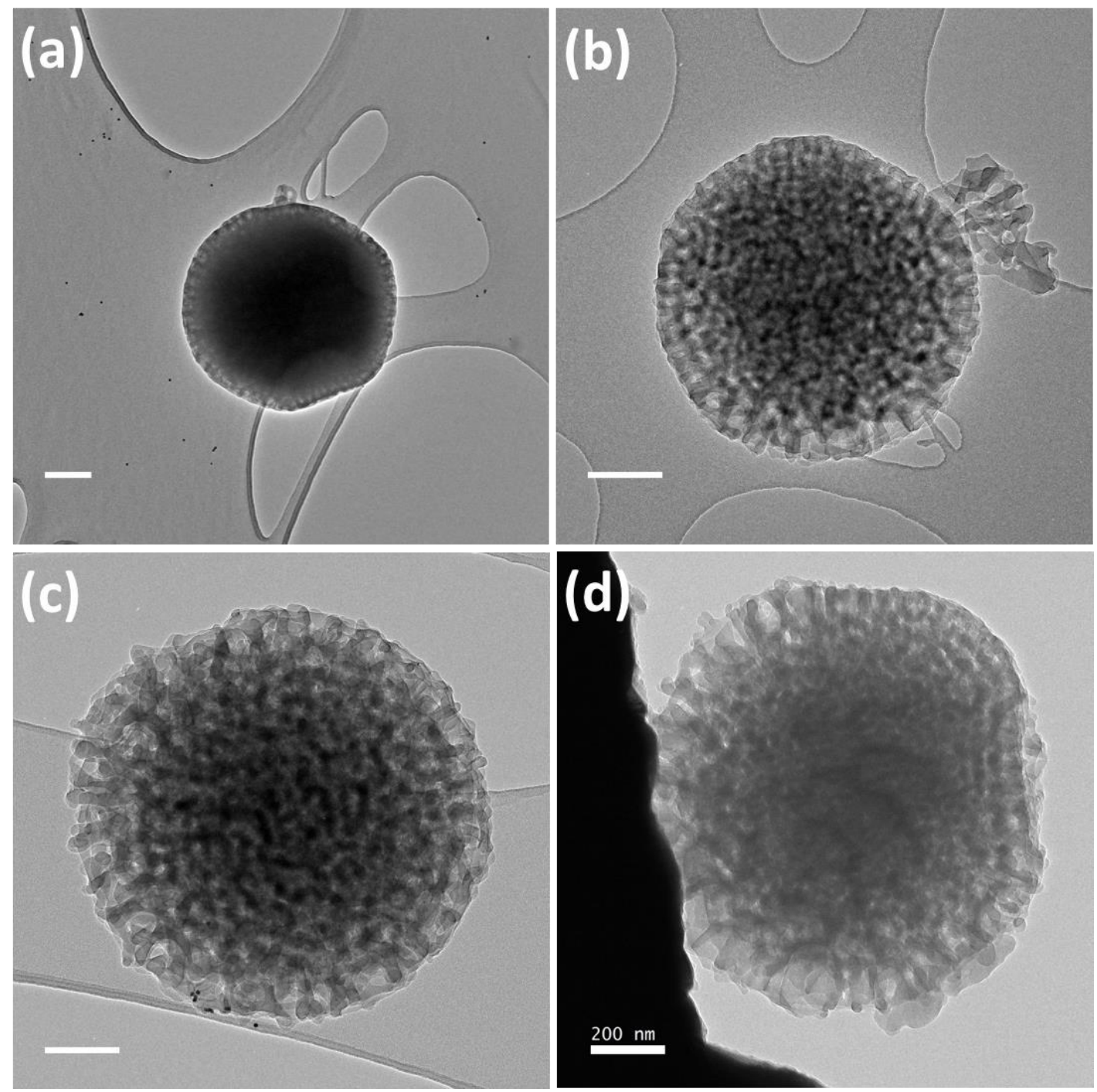

Figure 3. TEM bright-view images of PMMA- $b$-P4VP porous microparticles with varied P4VP molar fraction. (a) M-V13.3, (b) M-V19.9, (c) M-V27.8, (d) M-V30.9. TEM images show that porosity has penetrated through the whole particle in $\mathbf{b}, \mathbf{c}, \mathbf{d}$. Scale bar represents $200 \mathrm{~nm}$.

\section{Tilt TEM Tomography}

To further demonstrate the internal porosity, a 3D structural analysis of the pores throughout a whole microparticle was undertaken using tilt TEM imaging with angles of up to $120^{\circ}$. The particles initially displayed a very small shrinkage of $2-2.5 \%$ in diameter but then quickly stabilised under the conditions of the measurements being made. A certain level of radiation damage is to be expected upon extended exposure of the soft polymers to the electron beam, ${ }^{71}$ but it is clear that the lacy carbon film coated on the TEM grid was sufficient at dissipating the charge on the microparticles. 
Pore size measurements throughout the sample were made from the tomographic reconstruction in the $\mathrm{Z}$-axis (i.e. parallel with the original zero tilt image) to minimise distortion due to the missing wedge. Eight image slices (Fig. 4) for sample M-V30.9 reveal the pore structure. Reconstructed slices at the surface of the structure (Fig. 4, image 001,021$)$ reveal large open pores $\left(d_{\mathrm{w}}=70.8 \mathrm{~nm}\right)$. Slices in the middle of the reconstruction (passing though the centre of the microparticle) reveal much smaller pore widths at the particle centre $\left(d_{\mathrm{w}}=24.4 \mathrm{~nm}\right.$, image 141 in Fig. 4). All slices show the nanopore structures, which further confirm that the pores are developed throughout the particle.

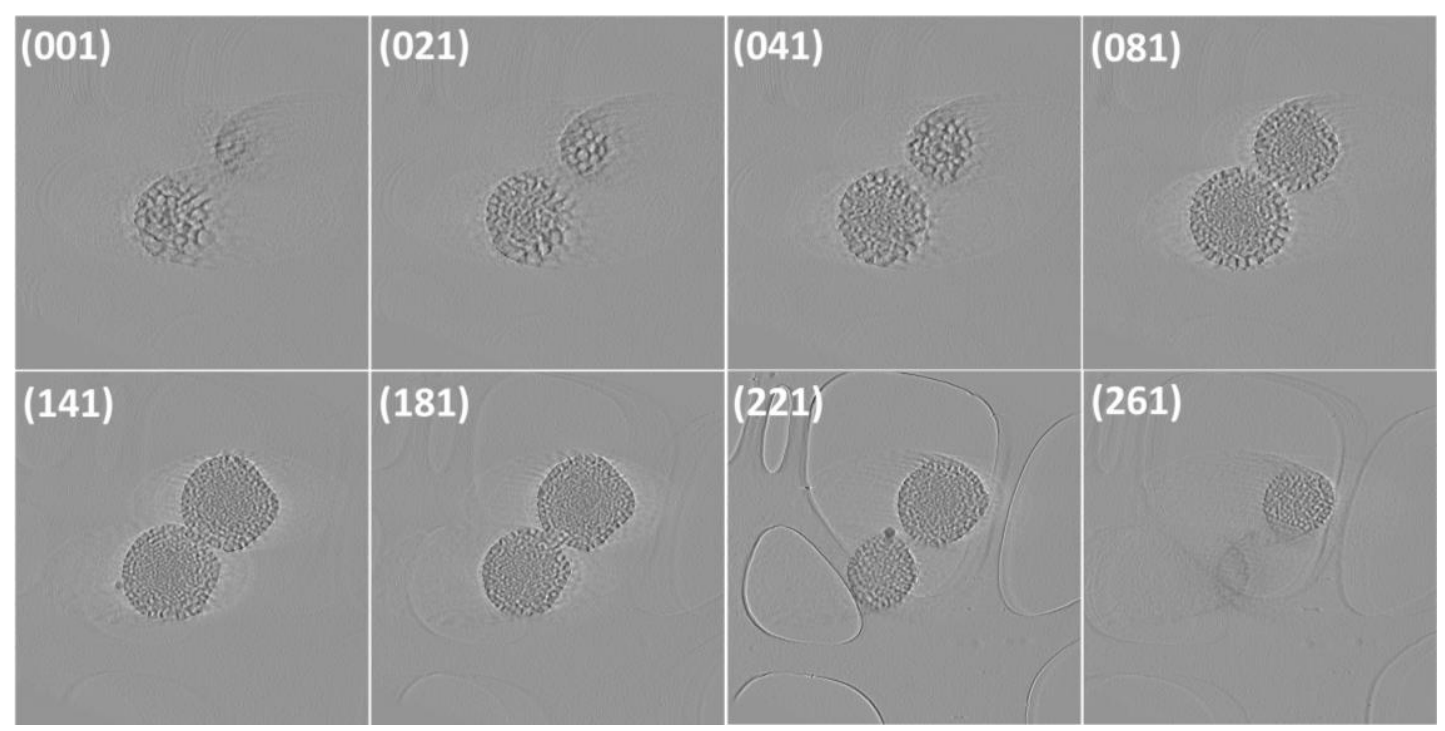

Figure 4. A selection of slices from the SIRT tomographic reconstruction of a porous microparticle (M-V30.9) showing 8 of the 280 slices from the top surface to the bottom and the supporting carbon film. The tilt series was taken at a 2550 electrons $/ \mathrm{nm}^{2} / \mathrm{s}$ dose rate with an 18 min and $41 \mathrm{~s}$ acquisition time.

The tilt series of images was then combined to form a video for each BCP sample with a different mol\% of P4VP (see illustrative videos in supplementary information). At low P4VP fraction (M-V13.3) isolated spherical pores have developed near the surface layers but the bulk appears to be nonporous (Fig. S7a). However, as the P4VP fraction increases it is clear that high quality internal porosity evolves with very clear interconnectivity throughout the entire particle (Fig. S7b-d).

The porous materials were further investigated using nitrogen adsorption isotherms (BET measurement at $-196{ }^{\circ} \mathrm{C}$, Fig. 5). The majority of the isotherms were typical type 
IV, characterised by the dominance of the $\mathrm{H} 1$ hysteresis loops observed at high relative pressures $\left(\mathrm{P} / \mathrm{P}^{0}\right)$ region. The adsorption capacity of nitrogen was negligible for virtually all the samples analysed at relative pressures up to 0.8 indicating that the materials are essentially purely mesoporous but containing some macropores. At low P4VP content, M-V13.3 presents a characteristic H2(b) loop, usually associated with "ink-bottle like" pores. But the porosity in this sample is quite low and consistent with the TEM/tilt TEM observations which reveal mesopores only in the sub-surface layers with pore width $21.2 \mathrm{~nm}$ (Fig 3a, Fig. S8a).

The other samples show greater porosity and pore size distributions (Fig. 5b, c) indicating that at higher mole ratios of P4VP there are multiple regular peaks of mesoand macropores centred at 25.2, 37.0, 50.3, 68.4, 86.2 and 117.2 nm. Such pore size distributions have previously only been observed for siliceous mesocellular foams with interconnected 3D pore cell structures, which can only be prepared by cumbersome hydrothermal templating methods under well-regulated and time-consuming conditions. 72,73 The characteristic surface textural properties of our materials appear to be dictated by the P4VP-block content, suggesting that the unique regular inter-connected 3D porous structures do originate from the geometric configurations of the block copolymer internal structures. TEM and Tilt TEM data again support our observations of surface and internal pores merging into longer channels and then interconnected pores (Figs. 3, 4).

The presence of micropores in these samples is small compared to the large population of meso- and macropores (Fig. S12). As a result, the specific BET surface areas of these materials are generally limited, ranging from 11 to $91 \mathrm{~m}^{2} / \mathrm{g}$ but with total pore volumes approaching up to $0.69 \mathrm{~cm}^{3} / \mathrm{g}$. However, what is clear is that the surface area and pore volume both increase significantly with increasing P4VP-content, demonstrating that this is the pore-generating domain during the swelling/de-swelling process. 

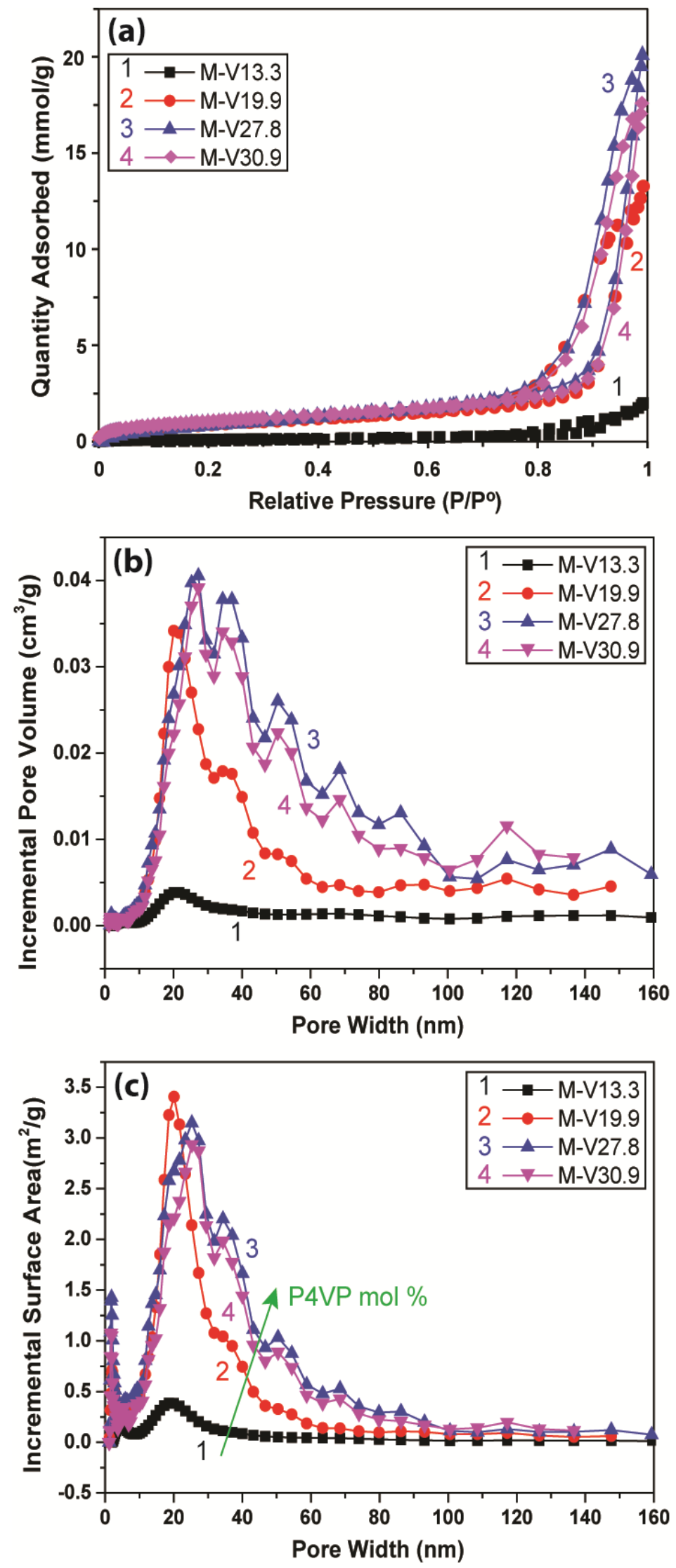

Figure 5. Nitrogen adsorption analysis of porous microparticles. (a) Hysteresis loop of Quantity Adsorbed versus Relative Pressure; (b) DFT pore volume distribution; and (c) DFT surface area distribution. 


\subsection{Pore size control by MW of BCP}

The size of the nanodomains formed via phase separation of the block copolymers is determined by the constituent block size, where the domain size is proportional to $N^{\mathrm{x}}$, with $N$ denoting the repeating unit and $\mathrm{x}<1 .{ }^{29,74}$ Here we demonstrate that RAFT controlled dispersion polymerisation in $\mathrm{scCO}_{2}$ can be utilised to achieve higher molecular weight blocks up to $330 \mathrm{kDa}$ (see Table 1). As expected, these much higher molecular weights lead to much larger domain sizes, with $\mathrm{d}(\mathrm{P} 4 \mathrm{VP})$ increasing from $25.5 \mathrm{~nm}$ to $60.4 \mathrm{~nm}$ as we progress from M-V30.9 (MW $=59400 \mathrm{~g} / \mathrm{mol}$ ) to the much larger M-V36.9 (MW $=153100 \mathrm{~g} / \mathrm{mol}$ ) (the as-synthesised domains are shown in Fig. S10). The swelling process for M-V36.9 yielded porous microparticles with isolated large pores at the particle surface (average pore width $d_{\mathrm{w}}=168.9 \mathrm{~nm}$ ) (Fig. 6). A TEM/tilt TEM video (Fig. S7e) clearly shows that the pores are interconnected and that these large pores are found at both the particle surface and internally (Fig. 6c). The absence of interparticle fusion for this system when compared with M-V36.4 (50k-25k) is attributed to the significantly reduced solubility of the P4VP block in ethanol as the molecular weight increases. Tomographic images based on the tilt TEM series show internal porosity (Fig. 6d) and demonstrate that the pore size can be controlled and extended to larger macropores of up to $200 \mathrm{~nm}$ by increasing the MW of the blocks, and hence also increasing the domain size and the available volume for ethanol swelling. Such large macropores cannot easily be achieved by conventional routes such as UV etching, ozonolysis, hydrolysis, or complexation with additives, which generally lead to much smaller mesopores $<50 \mathrm{~nm} .{ }^{25}$ In those methods the pore size is limited to the domain size of the intrinsic BCP self-assembled nanostructure, which is usually in the range of $10-100 \mathrm{~nm}$. By contrast, our approach using swelling induced order-to-order morphology transitions and morphology reconstruction by fast de-swelling can facilitate significantly larger features. 

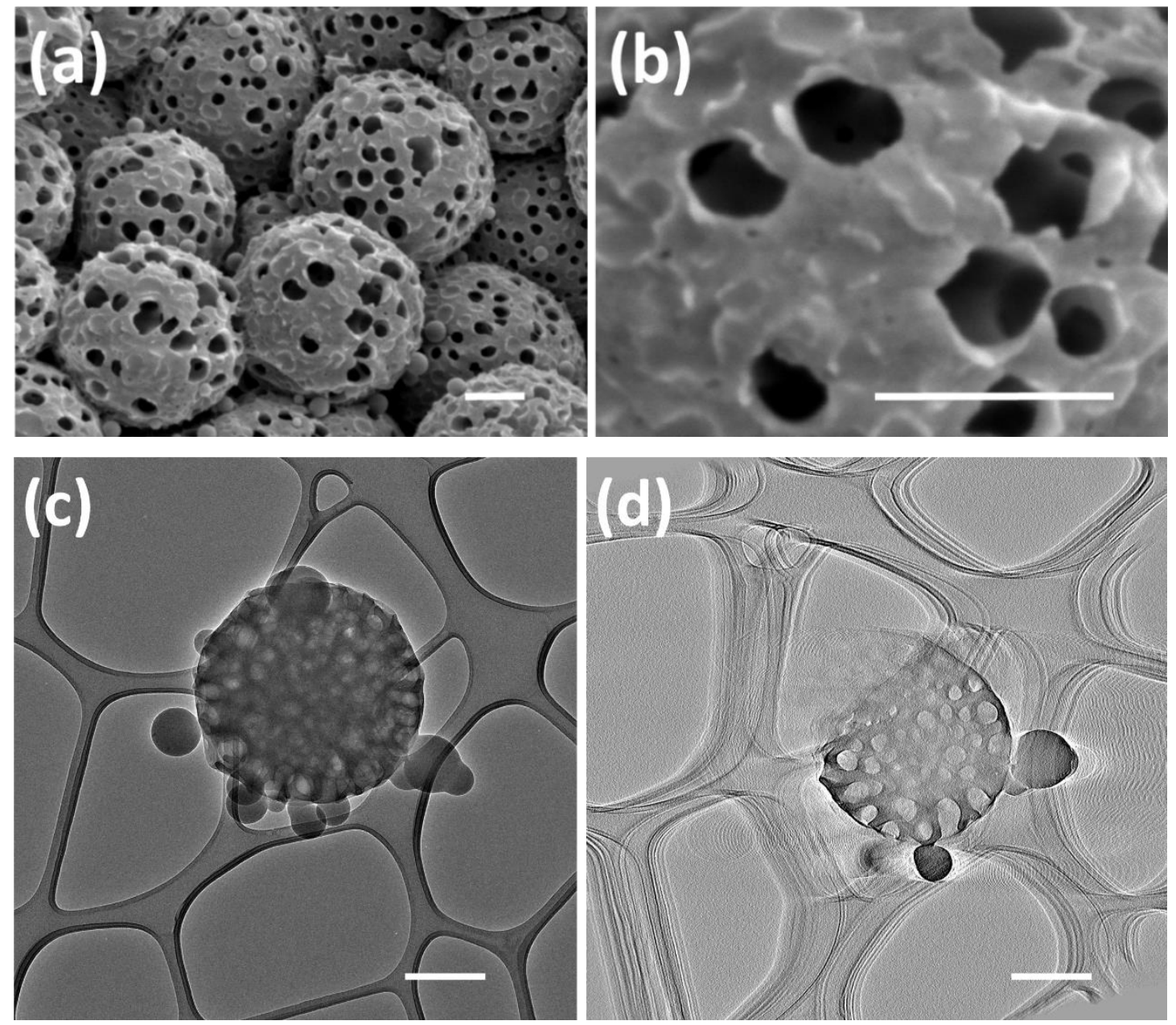

Figure 6. High molecular weight PMMA-b-P4VP porous microparticles M-V36.9 with block sizes targeted at 200k-133k. (a, b) SEM images at different magnification; (c) Bright-field TEM image and (d) a slice through the centre of the sample from a tomographic reconstruction (accelerating voltage of $200 \mathrm{kV}$; WBP reconstruction, no fiducial markers). Scale bar $500 \mathrm{~nm}$ in each case.

\section{Other block copolymer systems}

The same selective solvent swelling and fast de-swelling process was applied to a range of other microparticulate block copolymers. The key was to determine the most appropriate selective swelling solvent and de-swelling pair for other systems (Fig. 7) based upon PMMA- $b$-PDMA (20.2 mol\% PDMA, M-DMA20.2), and PMMA- $b$ PDMAEMA (11.8 mol\% PDMAEMA, M-DMAEMA11.8). The polymers were again synthesised via RAFT controlled dispersion polymerisation and treated with alcohols as a selective swelling solvent for the minority PDMA and PDMAEMA blocks and then hexane for de-swelling. The microparticulate morphology was well conserved for 
M-DMA20.2 (Fig. 7a,b) when swollen with ethanol compared to the original (Fig. S11g). The particles also developed surface open pores $\left(d_{\mathrm{w}}=26 \mathrm{~nm}\right)$ which appear interconnected. In contrast, swelling M-DMAEMA11.8 with ethanol causes interparticle fusion (Fig. S13). A lower alcohol of methanol was found to significantly reduce the particle fusion for M-DMAEMA11.8 (Fig. 7c,d). Surface open pores developed, but these were much smaller $d_{\mathrm{w}}=12 \mathrm{~nm}$. This illustrates that it is key to find the appropriate selective swelling solvent when changing the $\mathrm{BCP}$ system, by considering the solubility parameters of the swelling block/swelling solvent to achieve the optimal porous structures. The successful fabrication of nanoporous particles from PMMA- $b$-PDMA and PMMA- $b$-PDMAEMA highlights the versatility of our approach and could likely be applied to a very wide range of block copolymer systems to achieve nanoporous soft materials with bespoke surface functionality.
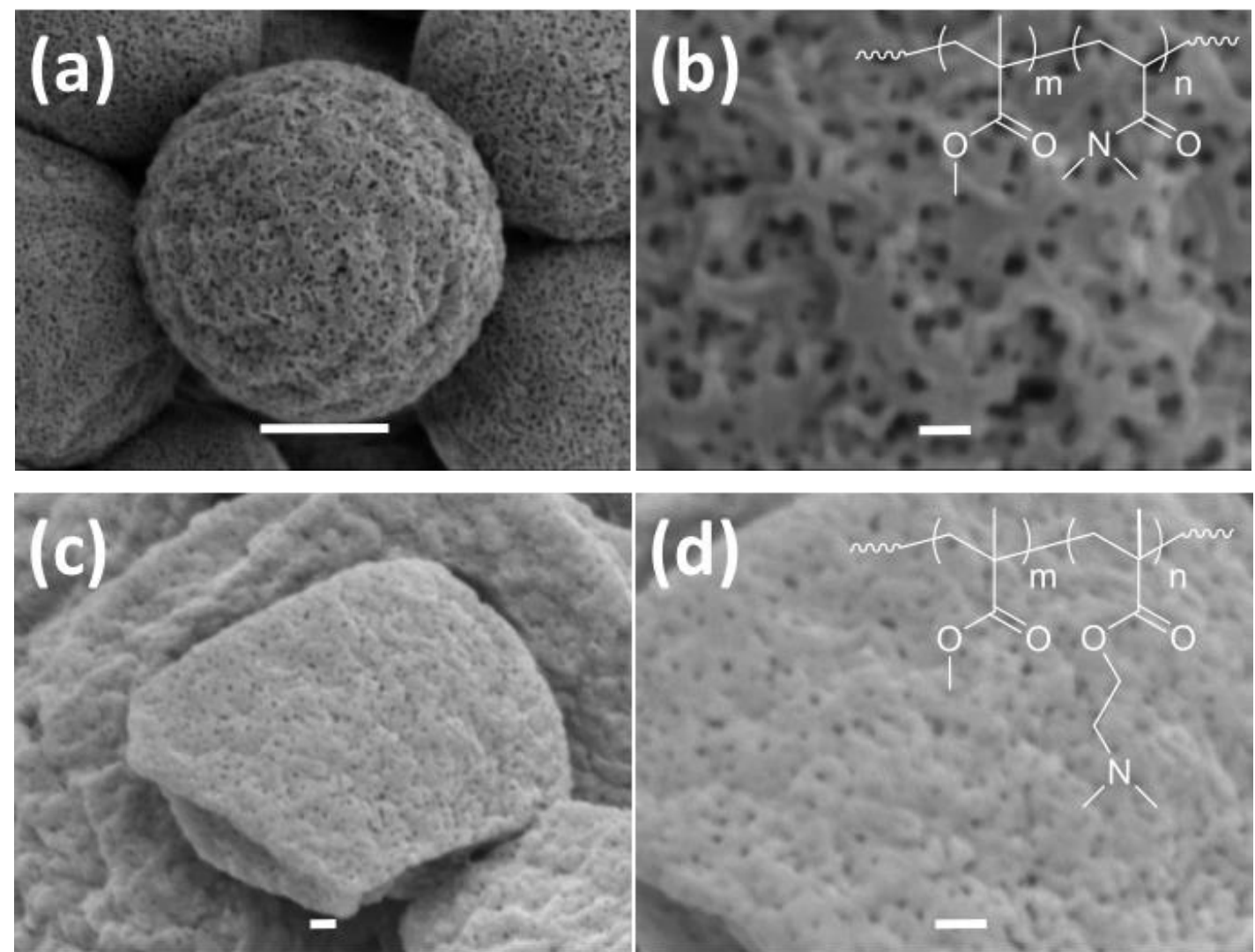

Figure 7. SEM images of PMMA- $b$-PDMA swollen with ethanol (a, b) and PMMA- $b$ PDMEAMA swollen with methanol (c, d) porous microparticles created by selective swelling in ethanol. (a, b) M-DMA20.2, (c, d) M-DMAEMA11.8. The inserts in (b, d) show the chemical structure of PMMA- $b$-PDMA and PMMA- $b$-PDMEAMA, respectively. Scale bar is $1000 \mathrm{~nm}$ in (a) and $100 \mathrm{~nm}$ in (b-d). 


\section{Conclusion}

We present a new methodology for creating controlled nanoporous block copolymer microparticles by synthesis in $\mathrm{scCO}_{2}$ followed by a swelling/rapid de-swelling route. Our use of a versatile RAFT controlled dispersion polymerisation in $\mathrm{scCO}_{2}$ allows excellent control of block lengths up to $330 \mathrm{kDa}$. Thus, we present fine control of the domain sizes in the BCP microparticles to provide a wide range of nanopore sizes and shapes. Increasing the length of the swellable block increases the swollen block volume and induces order-to-order morphology transitions in the swollen state. After rapid deswelling the resultant porosity shows a range of morphologies from isolated spheres through to short channels and interconnected networks; leading to unprecedentedly large pores $\sim 200 \mathrm{~nm}$. Innovative use of tilt-TEM microtomography has allowed us to probe in detail the structure of the porosity in the microparticles and to demonstrate definitively that the porosity persists throughout these larger 3-D structures. The method was also extended to a broader range of functional polymers including PMMA$b$-P4VP, PMMA- $b$-PDMA, and PMMA- $b$-PDMEAMA. Both the BCP microparticle synthesis and the swelling/rapid deswelling process have been demonstrated on the multigram scale and could easily be scaled to produce kilogram quantities. Thus, we have presented a facile, versatile and therefore practical strategy for the bespoke fabrication of hierarchically macro- and mesoporous block copolymer microparticles, with significant promise for use in a wide range of potential applications.

\section{Associated Content}

\section{Supporting Information}

Detailed BCP characteristics, GPC traces and SEM and TEM images of the original BCP microparticles before and/or after swelling. Tilt TEM videos demonstrating the fully porous nature of the five porous microparticle samples and their corresponding surface area and porosity properties. This material is available free of charge via the Internet at http://pubs.acs.org.

\section{Author Information}

\section{Corresponding Author}

steve.howdle@nottingham.ac.uk

\section{Notes}


The authors declare no competing financial interest.

\section{Acknowledgements}

We are grateful to the Leverhulme Trust (RPG-2014-034) for their support of this project (GH and TMB). MA is deeply grateful to the UNNES-IDB scholarship for a $\mathrm{PhD}$ Scholarship to Nottingham. STS is grateful to the Erasmus + scheme for the award of a higher education traineeship to support his visit to Nottingham. We thank the University of Nottingham and in particular the invaluable technical support received from our high pressure workshop technicians Richard Wilson, Peter Fields and Martin Dellar. Our research made use of the excellent electron microscopy facilities in the Nano-Micro Research Centre (NMRC) in the University of Nottingham in particular the 2100Plus TEM is supported by the EPSRC [EP/L022494/1]. We thank also Nicola Weston for the assistance in electron microscopies. We thank Yaoming Zhang for the valuable discussion/suggestions on P4VP and Prof Ullrich Steiner for his input on block copolymer materials.

\section{References}

(1) Barbaro, P.; Liguori, F. Chem. Rev. 2009, 109, 515-29.

(2) Sherrington, D. C. J. Polym. Sci. Part A: Polym. Chem. 2001, 39, 2364-2377.

(3) Say, R.; Emir, S.; Garipcan, B.; Patir, S. 1.; Denizli, A. Adv. Polym. Technol. 2003, 22, 355364.

(4) Bogdan, A. R.; Mason, B. P.; Sylvester, K. T.; McQuade, D. T. Angew. Chem. Int. Ed. 2007, 46, 1698-701.

(5) Lu, J.; Toy, P. H. Chem. Rev. 2009, 109, 815-38.

(6) Buchmeiser, M. R. Chem. Rev. 2009, 109, 303-21.

(7) Deng, J.; Yu, Y.; Dun, S.; Yang, W. J. Phys. Chem. B 2010, 114, 2593-601.

(8) Benes, M. J.; Horak, D.; Svec, F. J. Sep. Sci. 2005, 28, 1855-75.

(9) Haginaka, J. J. Chromatogr. B, Analyt. Technol. Biomed. Life. Sci. 2008, 866, 3-13.

(10) Basso, A.; Braiuca, P.; De Martin, L.; Ebert, C.; Gardossi, L.; Linda, P.; Verdelli, S.; Tam, A. Chemistry 2004, 10, 1007-13.

(11) Sinigoi, L.; Bravin, P.; Ebert, C.; D'Amelio, N.; Vaccari, L.; Ciccarelli, L.; Cantone, S.; Basso, A.; Gardossi, L. J. Comb. Chem. 2009, 11, 835-45.

(12) Mei, S.; Jafta, C. J.; Lauermann, I.; Ran, Q.; Kärgell, M.; Ballauff, M.; Lu, Y. Adv. Funct. Mater. 2017, 27.

(13) Wang, L.; Mei, S.; Jin, Z. Macromol. Chem. Phys. 2013, 214, 2579-2583. 
(14) Stein, A. Adv. Mater. 2003, 15, 763-775.

(15) Tokarev, I.; Minko, S. Adv. Mater. 2010, 22, 3446-62.

(16) Elbert, D. L. Acta. Biomater. 2011, 7, 31-56.

(17) Stich, M. I. J.; Schaeferling, M.; Wolfbeis, O. S. Adv. Mater. 2009, 21, 2216-2220.

(18) Salinas-Castillo, A.; Camprubi-Robles, M.; Mallavia, R. Chem. Commun. 2010, 46, 1263 5 .

(19) Lan, Y.; Yang, L.; Zhang, M.; Zhang, W.; Wang, S. ACS Appl. Mater. Interfaces 2010, 2, 127-33.

(20) Jiang, Y.; Sun, Q.; Zhang, L.; Jiang, Z. J. Mater. Chem. 2009, 19.

(21) Rastogi, V.; Velikov, K. P.; Velev, O. D. Phys. Chem. Chem. Phys. 2010, 12, 11975-83.

(22) Gokmen, M. T.; Du Prez, F. E. Prog. Polym. Sci. 2012, 37, 365-405.

(23) Guo, S.; Rzayev, J.; Bailey, T. S.; Zalusky, A. S.; Olayo-Valles, R.; Hillmyer, M. A. Chem. Mater. 2006, 18, 1719-1721.

(24) Bang, J.; Kim, S. H.; Drockenmuller, E.; Misner, M. J.; Russell, T. P.; Hawker, C. J. J. Am. Chem. Soc. 2006, 128, 7622-9.

(25) Wu, D.; Xu, F.; Sun, B.; Fu, R.; He, H.; Matyjaszewski, K. Chem. Rev. 2012, 112, 39594015.

(26) Mai, Y.; Eisenberg, A. Chem. Soc. Rev. 2012, 41, 5969-85.

(27) Hsueh, H. Y.; Yao, C. T.; Ho, R. M. Chem. Soc. Rev. 2015, 44, 1974-2018.

(28) Robbins, S. W.; Beaucage, P. A.; Sai, H.; Tan, K. W.; Werner, J. G.; Sethna, J. P.; DiSalvo, F. J.; Gruner, S. M.; Van Dover, R. B.; Wiesner, U. Sci. Adv. 2016, 2, e1501119.

(29) Bates, F. S.; Fredrickson, G. H. Annu. Rev. Phys. Chem. 1990, 41, 525-57.

(30) Hamley, I. W. Prog. Polym. Sci. 2009, 34, 1161-1210.

(31) Lynd, N. A.; Meuler, A. J.; Hillmyer, M. A. Prog. Polym. Sci. 2008, 33, 875-893.

(32) Abetz, V.; Simon, P. F. W., Phase Behaviour and Morphologies of Block Copolymers. In Block Copolymers I, Abetz, V., Ed. Springer Berlin Heidelberg: Berlin, Heidelberg, 2005; pp 125-212.

(33) Lee, J. S.; Hirao, A.; Nakahama, S. Macromolecules 1988, 21, 274-276.

(34) Bandyopadhyay, K.; Tan, E.; Ho, L.; Bundick, S.; Baker, S. M.; Niemz, A. Langmuir 2006, 22, 4978-84.

(35) Fu, G. D.; Yuan, Z.; Kang, E. T.; Neoh, K. G.; Lai, D. M.; Huan, A. C. H. Adv. Funct. Mater. 2005, 15, 315-322.

(36) Hedrick, J. L.; Carter, K. R.; Labadie, J. W.; Miller, R. D.; Volksen, W.; Hawker, C. J.; Yoon, D. Y.; Russell, T. P.; McGrath, J. E.; Briber, R. M. Adv. Polym. Sci. 1999, 141, 1-43.

(37) Chung, C.-M.; Lee, J.-H.; Cho, S.-Y.; Kim, J.-G.; Moon, S.-Y. J. Appl. Polym. Sci. 2006, 101, 532-538. 
(38) Lu, S.-Y.; Chang, C.-H.; Yu, C.-H.; Chen, H.-L.; Lo, Y. H. J. Mater. Res. 2011, 20, $1523-$ 1528.

(39) Olayo-Valles, R.; Guo, S.; Lund, M. S.; Leighton, C.; Hillmyer, M. A. Macromolecules 2005, 38, 10101-10108.

(40) Boontongkong, Y.; Cohen, R. E. Macromolecules 2002, 35, 3647-3652.

(41) Xu, C.; Fu, X.; Fryd, M.; Xu, S.; Wayland, B. B.; Winey, K. I.; Composto, R. J. Nano. Lett. 2006, 6, 282-7.

(42) Xu, C.; Wayland, B. B.; Fryd, M.; Winey, K. I.; Composto, R. J. Macromolecules 2006, 39, 6063-6070.

(43) Cho, H.; Park, H.; Russell, T. P.; Park, S. J. Mater. Chem. 2010, 20.

(44) Zettl, U.; Knoll, A.; Tsarkova, L. Langmuir 2010, 26, 6610-6617.

(45) Wang, L.; Montagne, F.; Hoffmann, P.; Pugin, R. Chem. Commun. 2009.

(46) Wang, Y.; Tong, L.; Steinhart, M. ACS Nano 2011, 5, 1928-38.

(47) Chai, J.; Buriak, J. M. ACS Nano 2008, 2, 489-501.

(48) Wang, Y.; Gösele, U.; Steinhart, M. Nano. Lett. 2008, 8, 3548-3553.

(49) Park, S.; Wang, J. Y.; Kim, B.; Russell, T. P. Nano. Lett. 2008, 8, 1667-72.

(50) Hwang, W.; Ham, M.-H.; Sohn, B.-H.; Huh, J.; Kang, Y. S.; Jeong, W.; Myoung, J.-M.; Park, C. Nanotechnology 2005, 16, 2897-2902.

(51) Park, S.; Wang, J.-Y.; Kim, B.; Xu, J.; Russell, T. P. ACS Nano 2008, 2, 766-772.

(52) Chen, Z.; He, C.; Li, F.; Tong, L.; Liao, X.; Wang, Y. Langmuir 2010, 26, 8869-74.

(53) Chen, J.-T.; Zhang, M.; Yang, L.; Collins, M.; Parks, J.; Avallone, A.; Russell, T. P. J. Polym. Sci. Part B: Polym. Phys. 2007, 45, 2912-2917.

(54) Wang, Y.; Gosele, U.; Steinhart, M. Nano. Lett. 2008, 8, 3548-53.

(55) Wang, Y.; Qin, Y.; Berger, A.; Yau, E.; He, C.; Zhang, L.; Gösele, U.; Knez, M.; Steinhart, M. Adv. Mater. 2009, 21, 2763-2766.

(56) Mei, S.; Feng, X.; Jin, Z. Soft Matter 2013, 9, 945-951.

(57) Chang, C.-W.; Chi, M.-H.; Ko, H.-W.; Chu, C.-W.; Fang, Z.-X.; Tu, Y.-H.; Chen, J.-T. Polym. Chem. 2017, 8, 3399-3404.

(58) Yin, J.; Yao, X.; Liou, J. Y.; Sun, W.; Sun, Y. S.; Wang, Y. ACS Nano 2013, 7, 9961-74.

(59) Wang, Y. Acc. Chem. Res. 2016, 49, 1401-1408.

(60) Mei, S.; Jin, Z. Small 2013, 9, 322-329.

(61) Fan, H.; Jin, Z. Macromolecules 2014, 47, 2674-2681.

(62) Ku, K. H.; Shin, J. M.; Klinger, D.; Jang, S. G.; Hayward, R. C.; Hawker, C. J.; Kim, B. J. ACS Nano 2016, 10, 5243-5251.

(63) Jennings, J.; Beija, M.; Richez, A. P.; Cooper, S. D.; Mignot, P. E.; Thurecht, K. J.; Jack, K. S.; Howdle, S. M. J. Am. Chem. Soc. 2012, 134, 4772-4781. 
(64) Jennings, J.; Beija, M.; Kennon, J. T.; Willcock, H.; O’Reilly, R. K.; Rimmer, S.; Howdle, S. M. Macromolecules 2013, 46, 6843-6851.

(65) Jennings, J.; Bassett, S. P.; Hermida-Merino, D.; Portale, G.; Bras, W.; Knight, L.; Titman, J. J.; Higuchi, T.; Jinnai, H.; Howdle, S. M. Polym. Chem 2016, 7, 905-916.

(66) McAllister, T. D.; Farrand, L. D.; Howdle, S. M. Macromol. Chem. Phys. 2016, 217, 22942301.

(67) Lai, J. T.; Filla, D.; Shea, R. Macromolecules 2002, 35, 6754-6756.

(68) Gregory, A. M.; Thurecht, K. J.; Howdle, S. M. Macromolecules 2008, 41, 1215-1222.

(69) Ren, Y.; Ma, Z.; Morris, R. E.; Liu, Z.; Jiao, F.; Dai, S.; Bruce, P. G. Nat. Commun. 2013, 4, 2015.

(70) Olivier, J. P. Carbon 1998, 36, 1469-1472.

(71) Egerton, R. F.; Li, P.; Malac, M. Micron 2004, 35, 399-409.

(72) Schmidt-Winkel, P.; Lukens, W. W.; Zhao, D.; Yang, P.; Chmelka, B. F.; Stucky, G. D. J. Am. Chem. Soc. 1999, 121, 254-255.

(73) Schmidt-Winkel, P.; Lukens, W. W.; Yang, P.; Margolese, D. I.; Lettow, J. S.; Ying, J. Y.; Stucky, G. D. Chem. Mater. 2000, 12, 686-696.

(74) Hamley, I. W., The Physics of Block Copolymers. Oxford University Press: 1998. 


\section{Supporting Information}
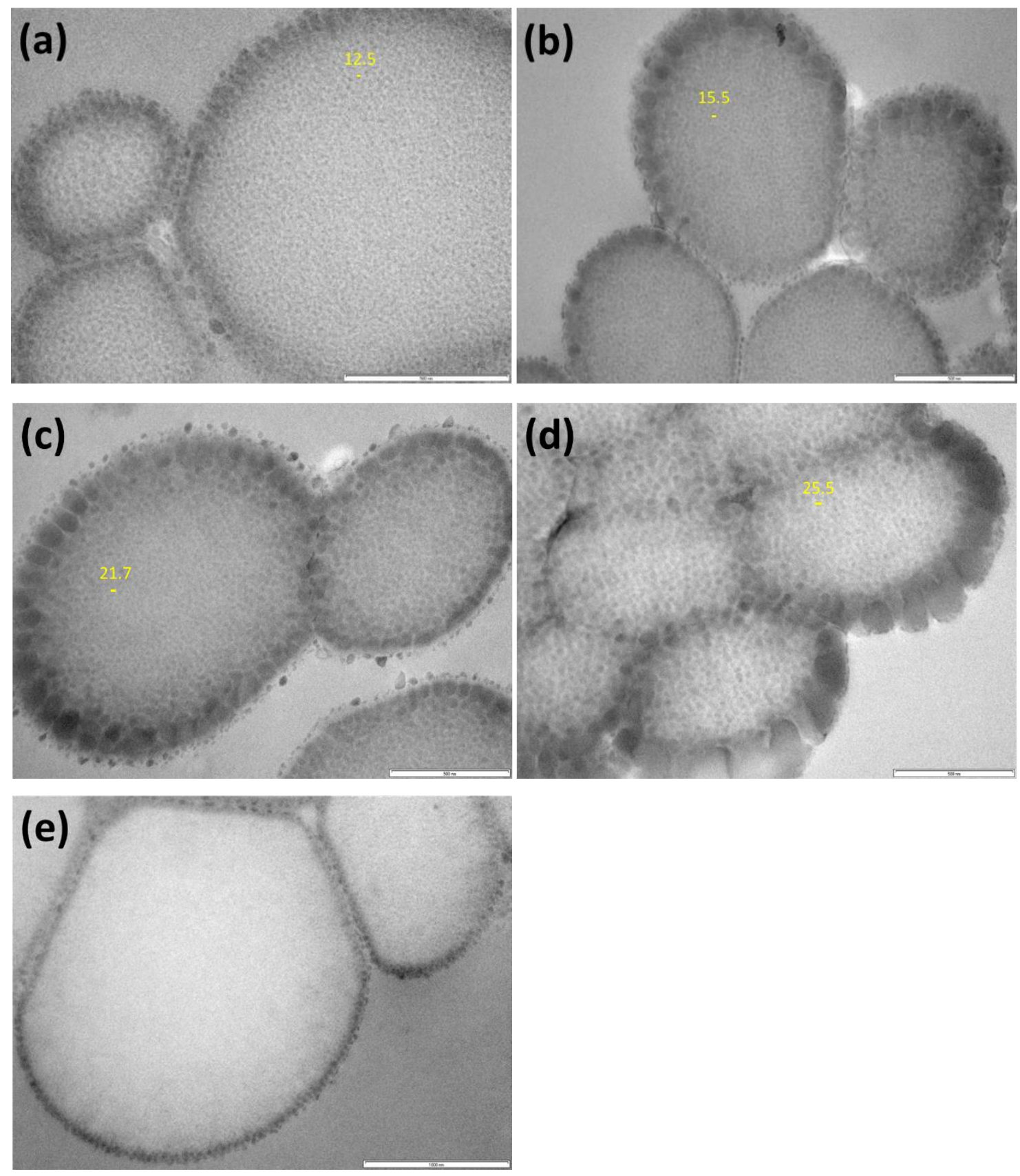

S1. TEM images of cross-sections ( $\sim 80 \mathrm{~nm}$ in thickness) from the original PMMA-b-P4VP microparticles demonstrating a spherical internal morphology and increasing domain sizes (ad). (a) M-V13.3, (b) M-V19.9, (c) M-V27.8, (d) M-V30.9, and the particles without internal structure (e) M-V9.1. The scale bar represents $500 \mathrm{~nm}$, except (e) with $1000 \mathrm{~nm}$. 
S2. Internal structures of original PMMA- $b-\mathrm{P} 4 \mathrm{VP}$ microparticles based on TEM analysis.

\begin{tabular}{llll}
\hline PMMA-P4VP & Morphology $^{\mathrm{a}}$ & \multicolumn{2}{c}{$\mathbf{d}^{\mathbf{P} 4 \mathrm{VP}} \mathbf{( \mathbf { n m } ) ^ { \mathrm { b } }}$} \\
\hline & & At centre & At periphery \\
\hline M-V9.1 & NON & -- & -- \\
\hline M-V13.3 & NON+SPH & $12.5 \pm 1.3$ & -- \\
\hline M-V19.9 & SPH (+coronal) & $15.5 \pm 1.6$ & $57.0 \pm 7.8$ \\
\hline M-V27.8 & SPH (+coronal) & $21.7 \pm 2.1$ & $60.9 \pm 12.6$ \\
\hline M-V30.9 & SPH (+coronal) & $25.5 \pm 2.2$ & $106.3 \pm 11.3$ \\
\hline M-V36.9 & SPH (+coronal) & $60.4 \pm 9.0$ & $116.4 \pm 7.3$
\end{tabular}

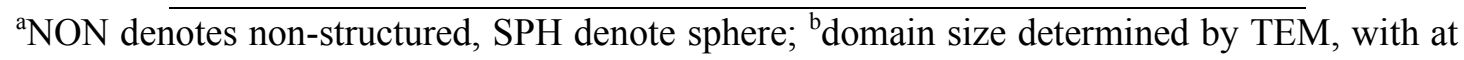
least 100 domains measured. 

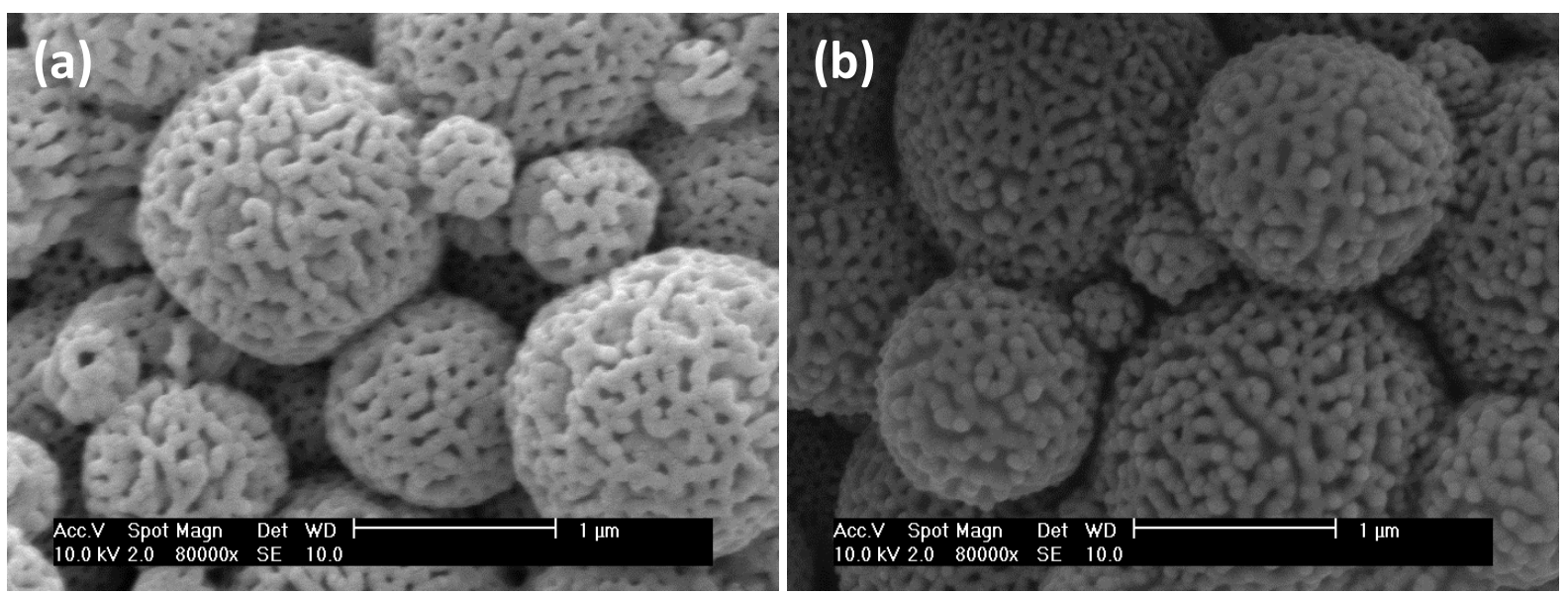

S3. (a) SEM image of the porous particles produced from the sample M-V27.8. (b) The same sample after storage in a glass vial on a bench at room temperature for approximately one year.

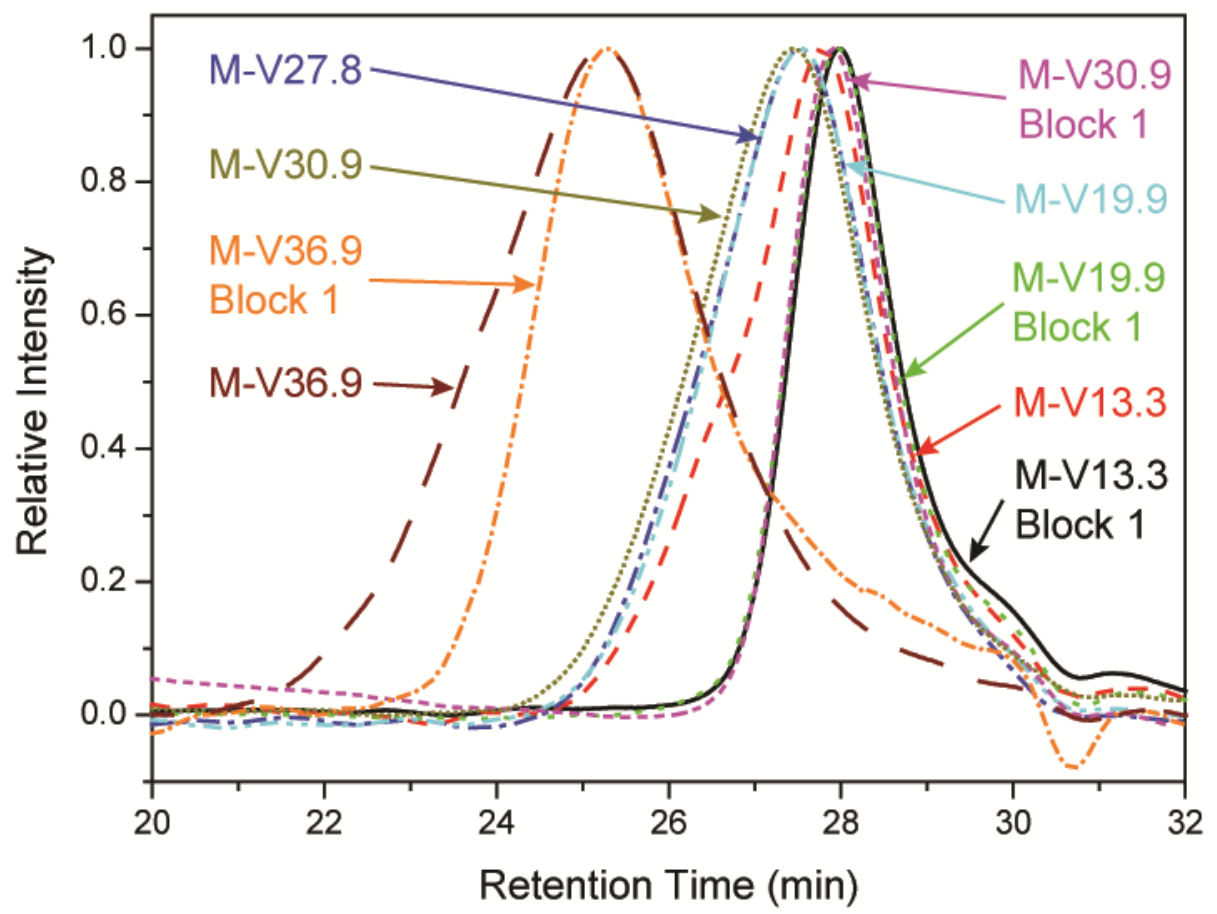

S4. GPC traces of the as-synthesised block copolymers listed in Table 1. Data for the reaction aliquots collected after the first PMMA block was polymerised but prior to injecting the second monomer are also included. 

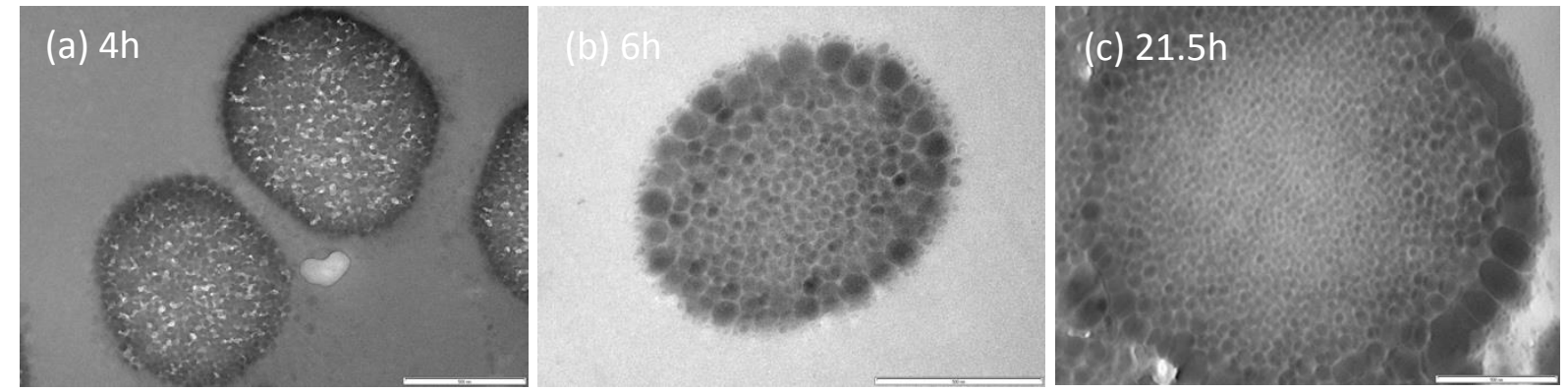

Fig. S5. TEM cross-sectional view of the sampling products during the chain extension of PMMA-P4VP (M-V41.2) at different time intervals. (a) $4 \mathrm{~h}$, (b) $6 \mathrm{~h}$ and (c) $21.5 \mathrm{~h}$.

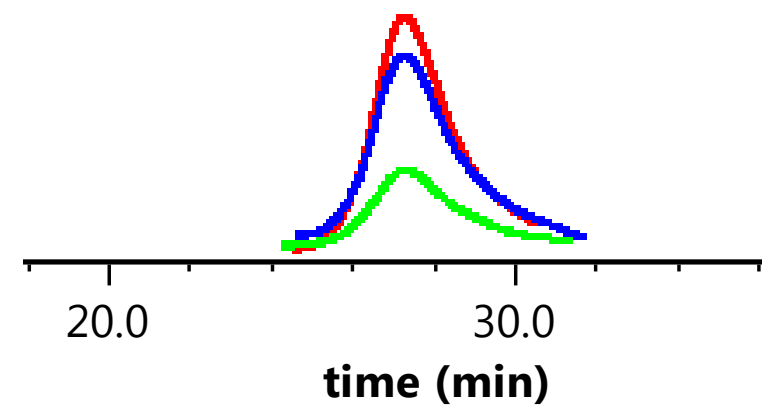

S6. GPC trace of sample M-V27.8 before (red) and after (blue and green) ethanol treatment. 
S7. Videos formed by Tilt TEM mapping of porous microparticles at varied tilt angels and each video is formed by $\sim 90$ TEM images at varied tilt angels with 1 degree increments. (a) M-V13.3, (b) M-V19.9, (c) M-V27.8, (d) M-V30.9, (e) M-V36.9.

\section{(Refer to video links)}
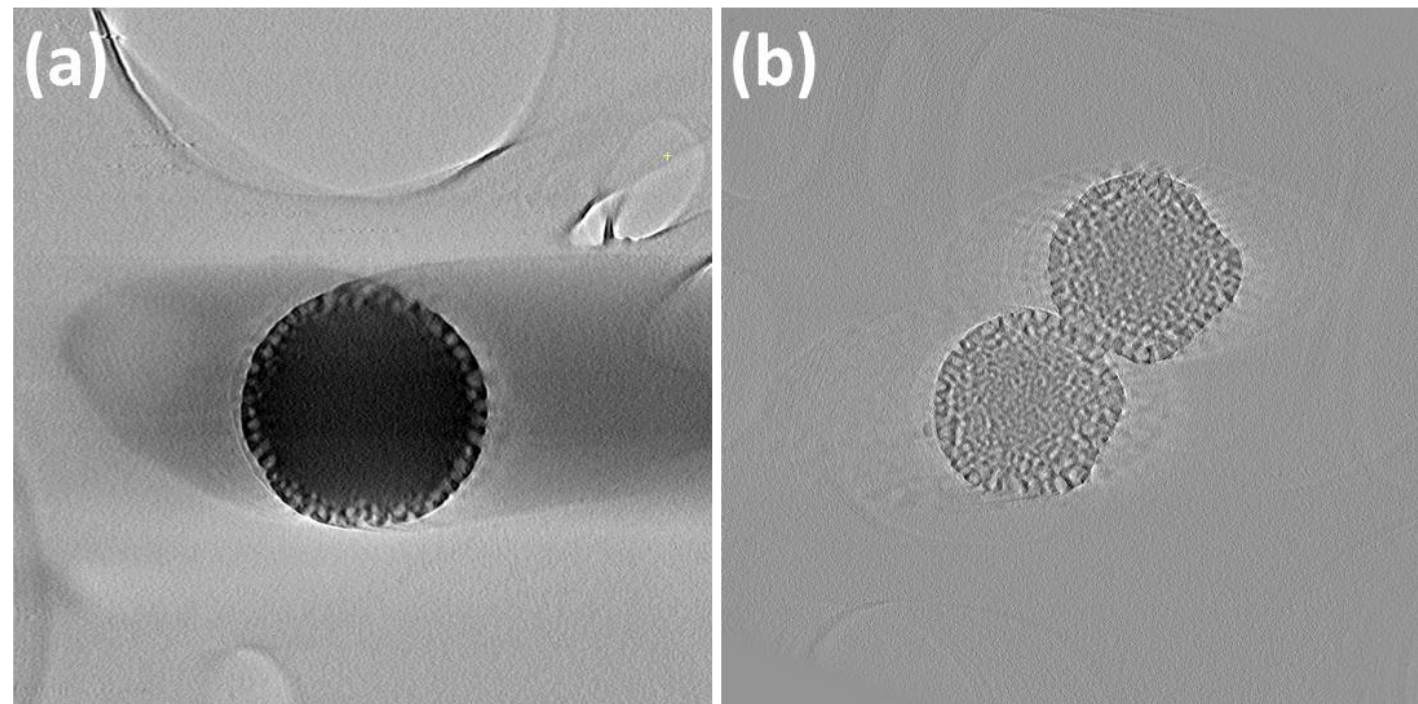

S8. 3D tomographic reconstruction of porous microparticles. A slice through near-centre from SIRT tomographic reconstruction of a porous microparticle from samples with varied P4VP mole fractions is presented. (a) M-V13.3, with pores only developed on the sub-surface layers, (b) M-V30.9, with pores that clearly have penetrated throughout the microparticle. 


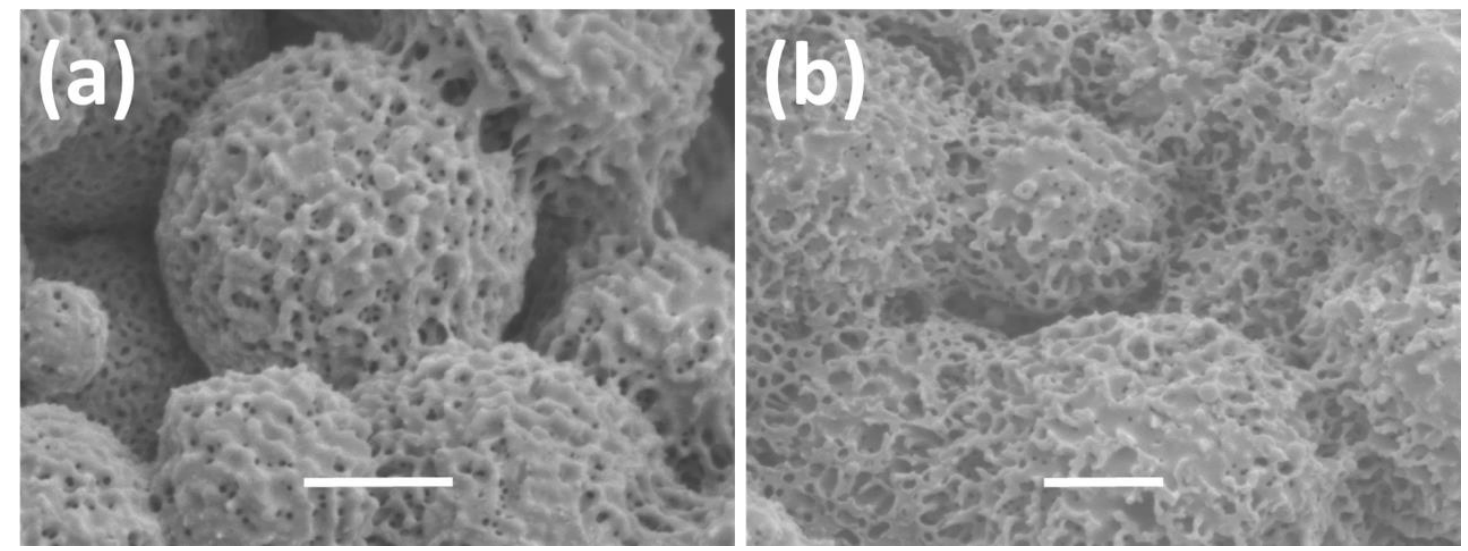

S9. SEM image of M-V36.4 demonstrating interparticle fusion at higher proprotions of P4VP. Scale bar is $250 \mathrm{~nm}$.
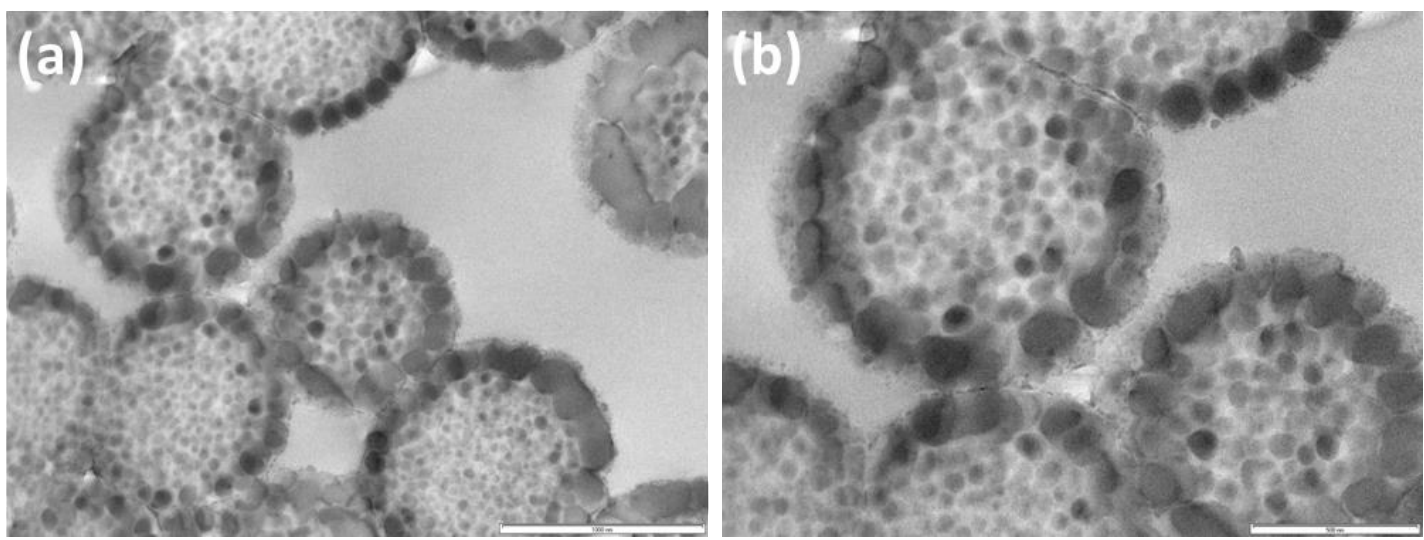

S10. TEM images of the cross-sections ( $~ 80 \mathrm{~nm}$ in thickness) of the original M-V36.9 (200kb-133k) microparticles. Scale bar is $1000 \mathrm{~nm}$ in (a) and $500 \mathrm{~nm}$ in (b).
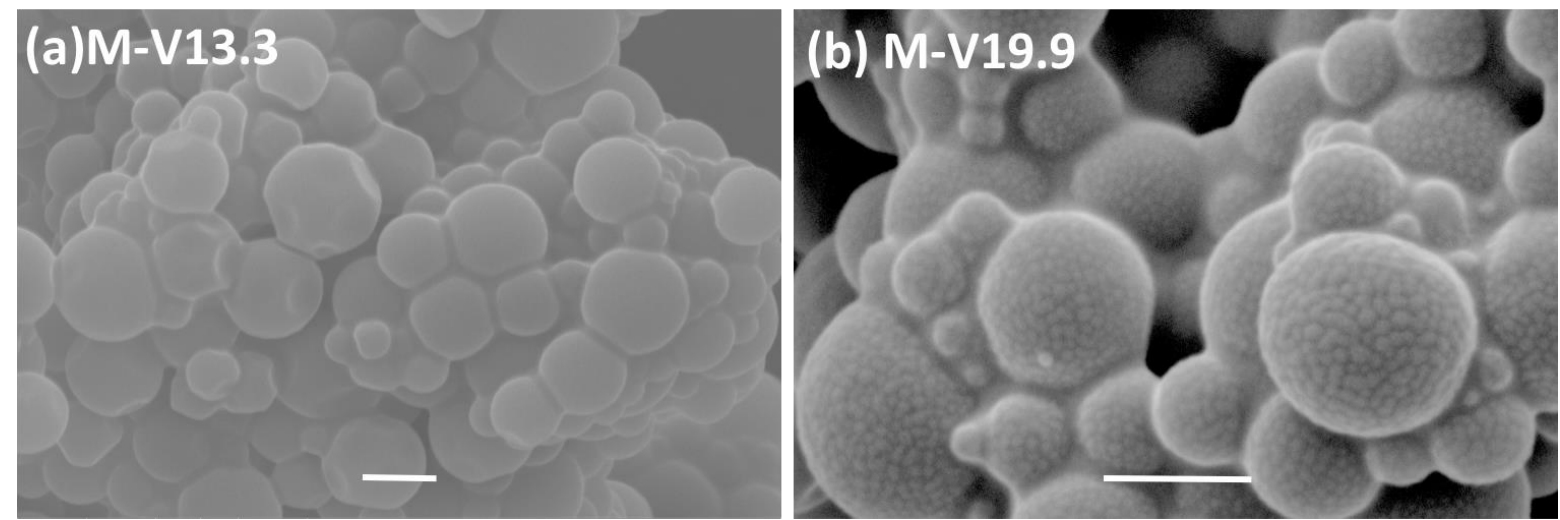

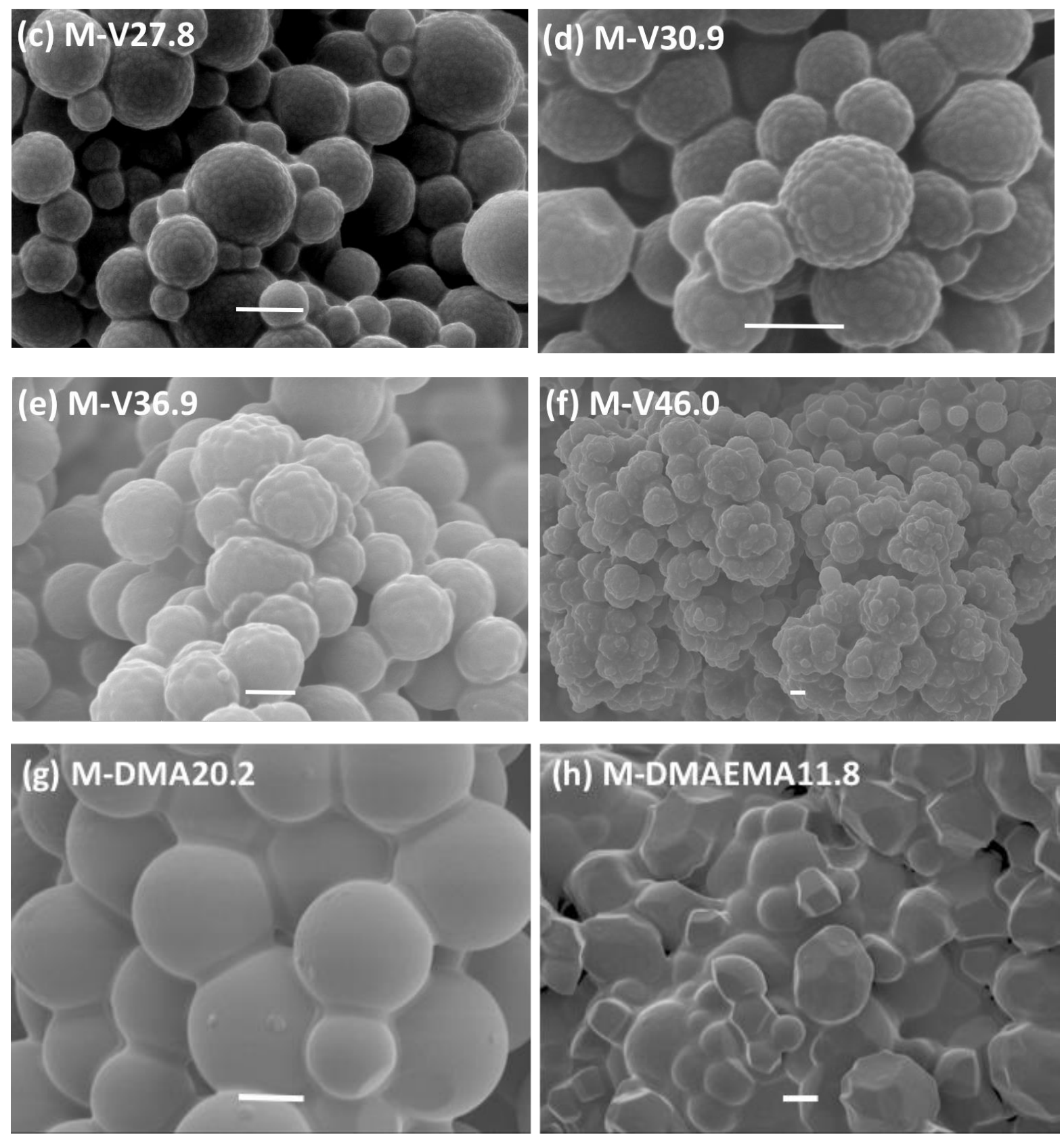

S11. SEM images of original microparticles prior to swelling/de-swelling, fabricated using RAFT dispersion polymerisation in $\mathrm{scCO}_{2}$. PMMA-b-P4VP microparticles (a) M-V13.3, (b) M-V19.9, (c) M-V27.8, (d) M-V30.9, (e) M-V36.9, (f) M-V46.0, (g) M-DMA20.2 (PMMA- $b$ PDMA) and (h) M-DMAEMA11.8 (PMMA- $b$-PDMAEMA). Scale bar $1000 \mathrm{~nm}$.

S12. Specific surface areas, pore structure parameters of the porous BCP microparticles.

\begin{tabular}{|c|c|c|c|c|c|c|c|}
\hline Samples & $\begin{array}{l}\mathrm{S}_{\mathrm{BET}} \\
\left(\mathrm{m}^{2} / \mathrm{g}\right)\end{array}$ & $\begin{array}{l}\mathrm{V}_{\text {total }} \\
\left(\mathrm{cm}^{3} / \mathrm{g}\right)\end{array}$ & $\begin{array}{l}\mathrm{V}_{\text {meso }} \\
\left(\mathrm{cm}^{3} / \mathrm{g}\right)\end{array}$ & $\begin{array}{l}V_{\text {micro }} \\
\left(\mathrm{cm}^{3} / \mathrm{g}\right)\end{array}$ & $\begin{array}{l}\text { Pore width }\left(\mathrm{N}_{2} \text { Ads }\right)^{\mathrm{a}} \\
\qquad(\mathrm{nm})\end{array}$ & $\begin{array}{l}\text { Pore width } \\
\text { (TEM) } \\
(\mathrm{nm})\end{array}$ & $\begin{array}{l}\text { Pore width } \\
\text { (SEM) } \\
(\mathrm{nm})\end{array}$ \\
\hline M-V13.3 & 11 & 0.069 & 0.052 & 0 & $20.1--\quad--\quad--\quad--$ & $21.2 \pm 1.7$ & $37.3 \pm 5.8$ \\
\hline
\end{tabular}




\begin{tabular}{|c|c|c|c|c|c|c|c|}
\hline M-V19.9 & 77.7 & 0.45 & 0.38 & 0.0029 & |21.6 $3750.3 \quad--$ & $40.2 \pm 6.1$ & $74.2 \pm 8.3$ \\
\hline M-V27.8 & 91.3 & 0.69 & 0.53 & 0.0017 & 25.23750 .368 .486 .2117 .2 & $54.5 \pm 8.9$ & $112.7 \pm 14.6$ \\
\hline M-V30.9 & 87.9 & 0.59 & 0.45 & 0.0038 & $25.23750 .3 \quad 68.486 .2 \quad 117.2$ & $85.8 \pm 16.4$ & $168.8 \pm 26.5$ \\
\hline
\end{tabular}

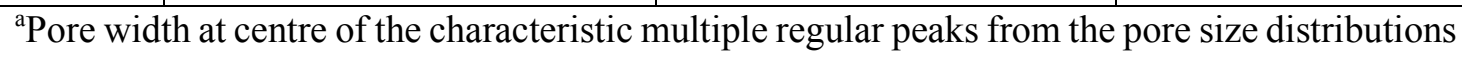
in Fig $\mathbf{5 b} ;{ }^{\text {b,c }}$ Surface pores.

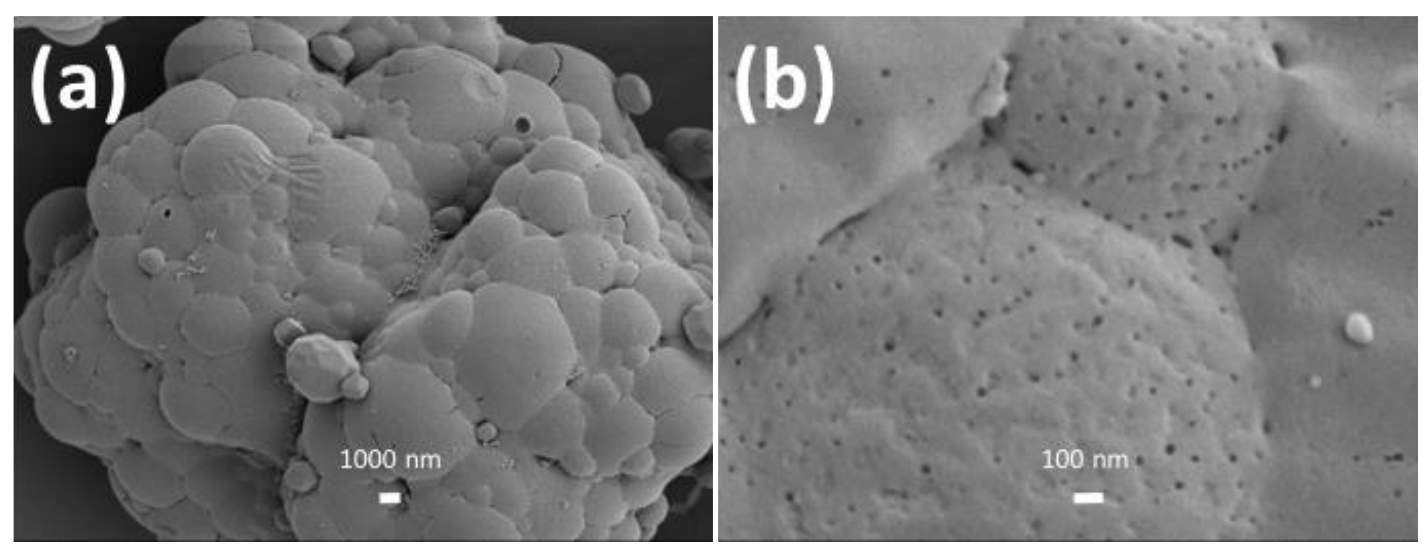

S13. SEM images of M-DMAEMA11.8 swollen with ethanol showing significant inter-particle fusion (a) and (b) at high magnification. 